\title{
Proton-neutron self-consistent quasiparticle random phase approximation within the $O(5)$ model
}

\author{
D. S. Delion \\ Institute of Physics and Nuclear Engineering, Bucharest Măgurele, POB MG-6, Romania \\ J. Dukelsky \\ Instituto de Estructura de la Materia, Serrano 123, E-28006 Madrid, Spain \\ P. Schuck \\ CNRS-IN2P3 Universite Joseph Fourier, Institut des Sciences Nucleaires, 53 Avenue des Martyrs, F-38026 Grenoble Cedex, France
}

E. J. V. de Passos

Instituto de Física, Universidade de São Paulo, C.P. 66318, 05389-970 São Paulo, Brazil

F. Krmpotic

Departamento de Física, Facultad de Ciencias Exactas, Universidad Nacional de La Plata, C. C. 67, 1900 La Plata, Argentina

(Received 22 March 2000; published 11 September 2000)

\begin{abstract}
The self-consistent quasiparticle random phase approximation (SCQRPA) within the $\mathrm{O}(5)$ model in the coupled proton-neutron representation is analyzed. The exact vacuum wave function is used to compute all involved matrix elements. A stability analysis of the stationary points is performed. A phase transition from the uncoupled to the coupled stable proton-neutron regime beyond the QRPA breakdown value of the particleparticle strength is evidenced. The excitation energies are close to the lowest stable exact eigenvalues given by the diagonalization procedure for all cases. The conditions for which the Ikeda sum rule is fulfilled for all values of the particle-particle strength are pointed out.
\end{abstract}

PACS number(s): 21.60.Jz, 23.40.Hc, 23.90.+w

\section{INTRODUCTION}

The renormalized quasiparticle random phase approximation (r-QRPA) became an important theoretical tool to investigate collective excitations in superfluid nuclei. In the last decade special attention was focused on applying this method to analyze the proton-neutron $(p n)$ interaction involved in beta and double beta decay processes [1]. Although the beta decay transition is modeled by a particlehole (p-h) operator, the important role of the particle-particle (p-p) channel in explaining the suppression of the twoneutrino double beta decay rate was evidenced by standard QRPA calculations [2]. Unfortunately the QRPA becomes unstable for physical values of the p-p interaction and this effect is connected with the overestimation of the ground state (g.s.) correlations. The r-QRPA accounts for g.s. correlation effects and is able to go a short distance beyond the transition point in a more or less reliable way [3-12]. The so-called self-consistent QRPA (SCQRPA) [13-17] accounts fully for the RPA ground state correlations and improves on the r-QRPA.

The importance of the $p n$ correlations in the GamowTeller double beta decay process was intensively investigated in the last decade $[18,19]$ and recently a critical analysis was performed in Ref. [20]. The $p n$ Hamiltonian can be diagonalized using different approximations. We mention here the most important methods: the use of a coupled $p n$ trial wave function [21], the $\alpha$-like representation of the quasiparticle as a creation proton operator plus the product of one neutron and two proton anihillation operators [22], and finally the most popular approach consisting in the general- ized $p n$ Bogoliubov transformation among one or more shells $[23,24]$. For a review of the properties and applications of this last transformation see, for instance, Ref. [25]. In order to test the accuracy of the different approximate methods several schematic $p n$ Hamiltonians were investigated [26-29].

In a recent paper [30] we made an analysis of the Fermi beta decay transitions in the $\mathrm{O}(5)$ model, using the exact SCQRPA vacuum wave function. As usual neutron-neutron $(n n)$ and proton-proton $(p p)$ pairing was assumed. The corresponding $u$ and $v$ amplitudes were obtained from the minimization of the SCQRPA ground state energy. Excitation energies and transition probabilities were obtained for values of the p-p interaction strength which are well above the point where standard QRPA breaks down. However, quantitative agreement with the results from exact diagonalization were rapidly degraded beyond the transition point. It is in fact well known from our studies with other models [15] that even with the SCRPA one has to change the single particle basis beyond the point where the standard RPA or, as in our case, the standard QRPA breaks down, which is where the first excited state becomes degenerate with the ground state. Since the RPA correlations which drive the system to the instability are, in the present context, $p n$ pair correlations, it is natural to augment the standard quasiparticle transformation to include mixing of proton and neutron states. We will show that in this new quasiparticle basis the standard QRPA has a physical branch beyond the transition point which continuously joins with the $p n$ uncoupled regime. We will extend the QRPA to the SCQRPA or r-QRPA and study excitation energies and transition probabilities in the region 
around and beyond the transition point.

The use of a quasiparticle basis including $p n$ mixing is, of course, not new and has also been applied within the context of double beta decay in a series of works $[18,19]$ using the standard QRPA. However, in these latter works only the region before and up to the transition point has been investigated. We therefore will apply for the first time the r-QRPA and SCQRPA in this context using this more general quasiparticle transformation and investigate the region beyond the transition point in a more systematic way. This is the main purpose of the present work.

In detail our paper is organized according to the following plan. In Sec. II is presented the $p n$ basis; in Sec. III is derived the self-consistent system of BCS equations, and the stability analysis of different solutions is performed. In Sec. IV the SCQRPA procedure is briefly described and in Sec. V the Ikeda sum rule is analyzed. Section VI is devoted to some representative numerical examples. Conclusions are drawn in the last section.

\section{EXTENDED QUASIPARTICLE BASIS}

Though the mixing of protons and neutrons in a generalized quasiparticle transformation is well documented in the literature [25] we here repeat the essential steps in order to make our paper self-contained and also to be able to connect this extended transformation to our more general SCQRPA (r-QRPA) approach.

Let us consider a spherical two-level $p n$ system with the same spin $j$. The general Bogoliubov transformation, connecting the particle $c_{\tau k}^{\dagger}(\tau=p, n)$ with the quasiparticle $b_{\mu k}^{\dagger}(\mu=1,2)$ representation, is given by the following relation [24]:

$$
\left(\begin{array}{c}
c_{p k}^{\dagger} \\
c_{n k}^{\dagger} \\
c_{p k} \bar{s}_{k} \\
c_{n k} \bar{s}_{k}
\end{array}\right)=\left(\begin{array}{cccc}
u_{1 p} & u_{2 p} & v_{1 p} & v_{2 p} \\
u_{1 n} & u_{2 n} & v_{1 n} & v_{2 n} \\
-v_{1 p} & -v_{2 p} & u_{1 p} & u_{2 p} \\
-v_{1 n} & -v_{2 n} & u_{1 n} & u_{2 n}
\end{array}\right)\left(\begin{array}{c}
b_{1 k}^{\dagger} \\
b_{2 k}^{\dagger} \\
b_{1 \bar{k}} s_{k} \\
b_{2 \bar{k}_{k}}
\end{array}\right),
$$

where $k$ is the spin projection and $s_{k} \equiv(-)^{j-k}$. This transformation mixes the proton with neutron states. In the case of complex amplitudes one can simultaneously analyze the isovector $(T=1)$ and isoscalar $(T=0)$ modes.

The interplay between the isoscalar and isovector pairing interactions is presently an important subject of investigation. The effect should be very important especially for $Z$ $\sim N$ nuclei. In Ref. [31] a realistic shell model calculation in the $p f$ shell evidenced a dominance of the isovector pairing in the ground state and the importance of the isoscalar component with increasing excitation energy. Recently in Ref. [32] an analysis of the interplay between isovector and isoscalar components within the proton-neutron coupled BCS approach for a $N=Z$ single level system was performed. A solution with a nonvanishing isoscalar gap was found, depending on the ratio between the isovector and isoscalar pairing strengths. A similar analysis, but within the $\mathrm{O}(8)$ model, is given by Ref. [33]. There the exact diagonalization proce- dure is compared with the BCS, QRPA, and r-QRPA results. The important role played by the isoscalar term in $\beta^{+}$transition and therefore in the double $\beta$ decay process is stressed.

In our analysis we will restrict ourselves to the isovector pairing interaction in order to investigate in the simplest case the idea that the changing to a "deformed" basis in the isospin space is the essential ingredient in restoring the stability of the system beyond the critical point. Therefore we will consider only real amplitudes in Eq. (2.1). A future inclusion of the isoscalar interaction within the $\mathrm{O}(8)$ model of Ref. [33] will of course improve our analysis. However, isoscalar pairing in any case can only be important and therefore influences the double beta decay for $N \sim Z$ nuclei.

A useful representation of the BCS amplitudes can be written in terms of the trigonometric functions [23]. It automatically satisfies the orthonormality relations and is a product of three rotations:

$$
\begin{aligned}
& \left(\begin{array}{cccc}
u_{1 p} & u_{2 p} & v_{1 p} & v_{2 p} \\
u_{1 n} & u_{2 n} & v_{1 n} & v_{2 n} \\
-v_{1 p} & -v_{2 p} & u_{1 p} & u_{2 p} \\
-v_{1 n} & -v_{2 n} & u_{1 n} & u_{2 n}
\end{array}\right) \\
& =\left(\begin{array}{cccc}
\cos \psi & \sin \psi & 0 & 0 \\
-\sin \psi & \cos \psi & 0 & 0 \\
0 & 0 & \cos \psi & \sin \psi \\
0 & 0 & -\sin \psi & \cos \psi
\end{array}\right) \\
& \times\left(\begin{array}{cccc}
\cos \alpha & 0 & \sin \alpha & 0 \\
0 & \cos \beta & 0 & \sin \beta \\
-\sin \alpha & 0 & \cos \alpha & 0 \\
0 & -\sin \beta & 0 & \cos \beta
\end{array}\right) \\
& \times\left(\begin{array}{cccc}
\cos \phi & \sin \phi & 0 & 0 \\
-\sin \phi & \cos \phi & 0 & 0 \\
0 & 0 & \cos \phi & \sin \phi \\
0 & 0 & -\sin \phi & \cos \phi
\end{array}\right) .
\end{aligned}
$$

This transformation has four independent parameters given by the angles characterizing the BCS amplitudes: $\alpha, \beta$ connecting the same kind of particles and $\psi, \phi$ which mixes the proton with the neutron states.

Let us introduce the number of particles and pairing operators in the particle representation,

$$
\begin{gathered}
N_{\tau_{1} \tau_{2}}=c_{\tau_{1} k}^{\dagger} c_{\tau_{2} k}, \quad \tau_{i}=p, n, \\
P_{\tau_{1} \tau_{2}}^{\dagger}=c_{\tau_{1} k}^{\dagger} c_{\tau_{2} k}^{\dagger} s_{k},
\end{gathered}
$$

and similar operators in the quasiparticle representation,

$$
\mathcal{N}_{i j}=b_{i k}^{+} b_{j k}, \quad i, j=1,2
$$


TABLE I. The coefficients of the operators entering the Hamiltonian (2.7) in the quasiparticle representation given by Eq. (2.9). $\bar{d}_{i j}=d_{i j} /\left(1+\delta_{i j}\right)=\sqrt{2 \Omega /\left(1+\delta_{i j}\right)}$.

\begin{tabular}{ccccccc}
\hline \hline$\gamma$ & $T_{\gamma}^{\dagger}$ & $x_{i j}^{\gamma}$ & $y_{i j}^{\gamma}$ & $z_{i j}^{\gamma}$ & $w^{\gamma}$ & $g_{\gamma}$ \\
\hline 1 & $N_{p}$ & $u_{i p} u_{j p}-v_{i p} v_{j p}$ & $\left(u_{i p} v_{j p}+u_{j p} v_{i p}\right) \overline{d_{i j}}$ & $\left(v_{i p} u_{j p}+v_{j p} u_{i p}\right) \bar{d}_{i j}$ & $2 \Omega \rho_{p p}$ & 0 \\
2 & $N_{n}$ & $u_{i n} u_{j n}-v_{i n} v_{j n}$ & $\left(u_{i n} v_{j n}+u_{j n} v_{i n}\right) \overline{d_{i j}}$ & $\left(v_{i n} u_{j n}+v_{j n} u_{i n}\right) \overline{d_{i j}}$ & $2 \Omega \rho_{n n}$ & 0 \\
3 & $N_{p n}$ & $u_{i p} u_{j n}-v_{i n} v_{j p}$ & $\left(u_{i p} v_{j n}+u_{j p} v_{i n}\right) \overline{d_{i j}}$ & $\left(v_{i p} u_{j n}+v_{j p} u_{i n}\right) \overline{d_{i j}}$ & $2 \Omega \rho_{p n}$ & $F_{p n} / 4$ \\
4 & $N_{n p}$ & $u_{i n} u_{j p}-v_{i p} v_{j n}$ & $\left(u_{i n} v_{j p}+u_{j n} v_{i p}\right) \overline{d_{i j}}$ & $\left(v_{i n} u_{j p}+v_{j n} u_{i p}\right) \overline{d_{i j}}$ & $2 \Omega \rho_{n p}$ & $F_{p n} / 4$ \\
5 & $P_{p}^{\dagger}$ & $-\left(u_{i p} v_{j p}+u_{i p} v_{j p}\right)$ & $\left(u_{i p} u_{j p}+u_{j p} u_{i p}\right) \bar{d}_{i j}$ & $-\left(v_{i p} v_{j p}+v_{j p} v_{i p}\right) \bar{d}_{i j}$ & $2 \Omega \chi_{p p}$ & $-G_{p} / 4$ \\
6 & $P_{n}^{\dagger}$ & $-\left(u_{i n} v_{j n}+u_{i n} v_{j n}\right)$ & $\left(u_{i n} u_{j n}+u_{j n} u_{i n}\right) \overline{d_{i j}}$ & $-\left(v_{i n} v_{j n}+v_{j n} v_{i n}\right) \overline{d_{i j}}$ & $2 \Omega \chi_{n n}$ & $-G_{n} / 4$ \\
7 & $P_{p n}^{\dagger}$ & $-\left(u_{i p} v_{j n}+u_{i n} v_{j p}\right)$ & $\left(u_{i p} u_{j n}+u_{j p} u_{i n}\right) \overline{d_{i j}}$ & $-\left(v_{i p} v_{j n}+v_{j p} v_{i n}\right) \overline{d_{i j}}$ & $2 \Omega \chi_{p n}$ & $-G_{p n} / 4$ \\
8 & $P_{n p}^{\dagger}$ & $-\left(u_{i n} v_{j p}+u_{i p} v_{j n}\right)$ & $\left(u_{i n} u_{j p}+u_{j n} u_{i p}\right) \overline{d_{i j}}$ & $-\left(v_{i n} v_{j p}+v_{j n} v_{i p}\right) \overline{d_{i j}}$ & $2 \Omega \chi_{n p}$ & $-G_{p n} / 4$ \\
\hline \hline
\end{tabular}

$$
\tilde{\mathcal{P}}_{i j}^{\dagger}=b_{i k}^{\dagger} b_{j k}^{\dagger} s_{k}=\tilde{\mathcal{P}}_{j i}^{\dagger} .
$$

Let us also introduce the normalized quasiparticle pair operators

$$
\mathcal{P}_{i j}^{\dagger}=\frac{\widetilde{\mathcal{P}}_{i j}^{\dagger}}{d_{i j}} ; \quad d_{i j}=\sqrt{2 \Omega\left(1+\delta_{i j}\right)},
$$

where $\Omega=j+\frac{1}{2}$. These operators satisfy the usual commutation relations within the $\mathrm{O}(5)$ algebra [26]. The commutators can be symbolically written as

$$
\begin{gathered}
{\left[\mathcal{P}_{i j}, \mathcal{P}_{k l}^{\dagger}\right]=\delta_{i j, k l}-a_{i j k l}^{m n} \mathcal{N}_{m n},} \\
{\left[\mathcal{N}_{i j}, \mathcal{P}_{k l}^{\dagger}\right]=b_{i j k l}^{m n} \mathcal{P}_{m n}^{\dagger},} \\
{\left[\mathcal{P}_{i j}, \mathcal{N}_{k l}\right]=c_{i j k l}^{m n} \mathcal{P}_{m n}, \quad c_{i j k l}^{m n}=b_{l k i j}^{m n},}
\end{gathered}
$$

where summation over the repeated labels is understood. This symbolic way to consider the commutation relations will help us to derive different relations in a more compact form.

We will use in our analysis the same Hamiltonian as in Refs. [16,30],

$$
\begin{aligned}
H= & \epsilon_{p} N_{p}+\epsilon_{n} N_{n}+\frac{F_{p n}}{4}\left(N_{p n} N_{n p}+N_{n p} N_{p n}\right) \\
& -\frac{G_{p}}{4} P_{p}^{\dagger} P_{p}-\frac{G_{n}}{4} P_{n}^{\dagger} P_{n}-\frac{G_{p n}}{4}\left(P_{p n}^{\dagger} P_{n p}+P_{n p}^{\dagger} P_{p n}\right) \\
\equiv & \epsilon_{\delta} T_{\delta}^{\dagger}+g_{\gamma} T_{\gamma}^{\dagger} T_{\gamma},
\end{aligned}
$$

where $\delta=1,2$ and $\gamma=3-8$ and

$$
\epsilon_{p}=e_{p}-\lambda_{p}, \quad \epsilon_{n}=e_{n}-\lambda_{n} .
$$

Here $e_{\tau}$ denotes the single particle energies and $\lambda_{\tau}$ Lagrange multipliers accounting for the number of particles conservation law. This Hamiltonian contains in addition to the proton and neutron pairing terms a p-h interaction with strength $F_{p n}$ and a p-p part with strength $G_{p n}$, which actually is the $p n$ pairing interaction. This schematic Hamiltonian is appropriate to simulate the important features of the monopole
(Fermi) beta decay process. The $p n$ quasiparticle representation of a one-body operator can be written as

$$
T_{\gamma}^{\dagger}=x_{i j}^{\gamma} \mathcal{N}_{i j}+y_{i j}^{\gamma} \mathcal{P}_{i j}^{\dagger}+z_{i j}^{\gamma} \mathcal{P}_{i j}+w^{\gamma},
$$

where the summation for $\mathcal{N}_{i j}$ operators is over $(i, j)$ $=(1,1),(2,2),(1,2),(2,1)$ and for $\mathcal{P}_{i j}$ operators over $(i, j)$ $=(1,1),(2,2),(1,2)$ indices. Here the index $\gamma$ corresponds to a concrete operator as in Table I.

For the normal and pairing densities, respectively,

$$
\begin{aligned}
& \rho_{\tau_{1} \tau_{2}}=v_{i \tau_{1}} v_{i \tau_{2}}, \\
& \chi_{\tau_{1} \tau_{2}}=v_{i \tau_{1}} u_{i \tau_{2}},
\end{aligned}
$$

by using the trigonometric representation of the BCS amplitudes (2.2), one obtains the following relations:

$$
\begin{gathered}
\rho_{p p}=\sin ^{2} \alpha \cos ^{2} \phi+\sin ^{2} \beta \sin ^{2} \phi, \\
\rho_{n n}=\sin ^{2} \alpha \sin ^{2} \phi+\sin ^{2} \beta \cos ^{2} \phi, \\
\rho_{p n}=\sin 2 \phi\left(\sin ^{2} \alpha-\sin ^{2} \beta\right) / 2, \\
\chi_{p p}=\left(\sin 2 \alpha \cos ^{2} \phi+\sin 2 \beta \sin ^{2} \phi\right) / 2, \\
\chi_{n n}=\left(\sin 2 \alpha \sin ^{2} \phi+\sin 2 \beta \cos ^{2} \phi\right) / 2, \\
\chi_{p n}=\sin 2 \phi(\sin 2 \alpha-\sin 2 \beta) / 4 .
\end{gathered}
$$

The Hamiltonian written in the quasiparticle representation takes the standard form

$$
H=H^{00}+H^{11}+H^{20}+H^{31}+H^{22}+H^{40},
$$

where the different $H^{i j}$ terms are given by

$$
\begin{array}{r}
H^{00}=\epsilon_{\delta} w^{\delta}+g_{\gamma}\left(w^{\gamma} w^{\gamma}+z_{m n}^{\gamma} z_{m n}^{\gamma}\right), \\
H^{11}=E_{m n} \mathcal{N}_{m n}, \\
H^{20}=\left(\epsilon_{\delta} z_{m n}^{\delta}+h_{m n}^{20}\right)\left(\mathcal{P}_{m n}^{\dagger}+\mathcal{P}_{m n}\right), \\
H^{31}=h_{m n k l}^{31}\left(\mathcal{P}_{m n}^{\dagger} \mathcal{N}_{l k}+\mathcal{N}_{k l} \mathcal{P}_{m n}\right),
\end{array}
$$




$$
\begin{gathered}
H^{22}=h_{m n k l}^{22} \mathcal{P}_{m n}^{\dagger} \mathcal{P}_{k l}+g_{m n k l}^{22}\left[\mathcal{N}_{m n} \mathcal{N}_{k l}-\delta_{n k} \mathcal{N}_{m l}\right] \\
H^{40}=h_{m n k l}^{40}\left(\mathcal{P}_{m n}^{\dagger} \mathcal{P}_{k l}^{\dagger}+\mathcal{P}_{m n} \mathcal{P}_{k l}\right) .
\end{gathered}
$$

The quasiparticle energies $E_{m n}$ are defined as the coefficients of $\mathcal{N}_{m n}$ :

$$
E_{m n}=\epsilon_{\delta} x_{m n}^{\delta}+g_{\gamma}\left[w^{\gamma}\left(x_{m n}^{\gamma}+x_{n m}^{\gamma}\right)-z_{i j}^{\gamma} z_{k l}^{\gamma} a_{i j k l}^{m n}\right]+g_{m k k n}^{22} .
$$

The other notation in Eqs. (2.13) is defined as follows:

$$
\begin{gathered}
h_{m n}^{20}=g_{\gamma}\left[w^{\gamma}\left(y_{m n}^{\gamma}+z_{m n}^{\gamma}\right)+z_{i j}^{\gamma} x_{k l}^{\gamma} b_{k l i j}^{m n}\right], \\
h_{m n k l}^{31}=g_{\gamma}\left(y_{m n}^{\gamma} x_{k l}^{\gamma}+z_{m n}^{\gamma} x_{l k}^{\gamma}\right), \\
h_{m n k l}^{22}=g_{\gamma}\left(y_{m n}^{\gamma} y_{k l}^{\gamma}+z_{m n}^{\gamma} z_{k l}^{\gamma}\right), \\
g_{m n k l}^{22}=g_{\gamma} x_{m n}^{\gamma} x_{l k}^{\gamma}, \\
h_{m n k l}^{40}=g_{\gamma} y_{m n}^{\gamma} z_{k l}^{\gamma},
\end{gathered}
$$

where the summation on the subscript indices is taken over $(1,1),(2,2),(1,2)$ in $y, z$ and over $(1,1),(2,2),(1,2),(2,1)$ in $E, x$ coefficients.

The expectation value of the Hamiltonian on the standard BCS wave function takes the following form in terms of densities:

$$
\begin{aligned}
H^{00}= & 2 \Omega \epsilon_{p}^{\prime} \rho_{p p}+2 \Omega \epsilon_{n}^{\prime} \rho_{n n}-G_{p}\left(\Omega \chi_{p p}\right)^{2}-G_{n}\left(\Omega \chi_{n n}\right)^{2} \\
& -2 G_{p n}^{\prime}\left(\Omega \chi_{p n}\right)^{2}+2 F_{p n}^{\prime}\left(\Omega \rho_{p n}\right)^{2}
\end{aligned}
$$

where the renormalized prime energies and strengths are defined by

$$
\begin{gathered}
\epsilon_{p}^{\prime}=\epsilon_{p}-\frac{G_{p}}{2} \rho_{p p}+\frac{F_{p n}}{4}-\frac{G_{p n}+F_{p n}}{4} \rho_{n n}, \\
\epsilon_{n}^{\prime}=\epsilon_{n}-\frac{G_{n}}{2} \rho_{n n}+\frac{F_{p n}}{4}-\frac{G_{p n}+F_{p n}}{4} \rho_{p p}, \\
G_{p n}^{\prime}=G_{p n}-\frac{1}{2 \Omega} F_{p n}, \\
F_{p n}^{\prime}=F_{p n}-\frac{1}{2 \Omega} G_{p n} .
\end{gathered}
$$

\section{SELF-CONSISTENT BCS EQUATIONS}

The generalized BCS equations within the SCQRPA scheme are derived from the extremum condition $\delta\langle H\rangle=0$ [34], i.e.,

$$
\begin{gathered}
\left\langle\left[H, \mathcal{P}_{a b}^{\dagger}\right]\right\rangle=\left\langle\left[H^{20}+H^{31}, \mathcal{P}_{a b}^{\dagger}\right]\right\rangle=0 \\
(a, b)=(1,1),(2,2),(1,2)
\end{gathered}
$$

Using the Hamiltonian written in the quasiparticle representation (2.13) one obtains

$$
\begin{gathered}
\left(z_{a b}^{\delta}-z_{m n}^{\delta} a_{m n a b}^{r s}\left\langle\mathcal{N}_{r s}\right\rangle\right) \epsilon_{\delta}+h_{a b}^{20}-h_{m n}^{20} a_{m n a b}^{r s}\left\langle\mathcal{N}_{r s}\right\rangle \\
+h_{a b k l}^{31}\left\langle\mathcal{N}_{k l}\right\rangle+h_{m n k l}^{31}\left(-a_{m n a b}^{r s}\left\langle\mathcal{N}_{k l} \mathcal{N}_{r s}\right\rangle\right. \\
\left.+b_{l k a b}^{r s}\left\langle\mathcal{P}_{m n}^{\dagger} \mathcal{P}_{r s}^{\dagger}\right\rangle+b_{k l a b}^{r s}\left\langle\mathcal{P}_{r s}^{\dagger} \mathcal{P}_{m n}\right\rangle\right)=0
\end{gathered}
$$

where the expectation value of an operator $T$ on the SCQRPA vacuum $|R P A\rangle$ is defined as

$$
\langle T\rangle \equiv \frac{\langle\mathrm{RPA}|T| \mathrm{RPA}\rangle}{\langle\mathrm{RPA} \mid \mathrm{RPA}\rangle} .
$$

The system given by Eq. (3.2) can be seen as a system of three linear equations for two unknowns $\epsilon_{1}$ and $\epsilon_{2}$ :

$$
D_{\alpha 1} \epsilon_{1}+D_{\alpha 2} \epsilon_{2}+D_{\alpha 3}=0, \quad \alpha \equiv(a, b) .
$$

In order to have a solution of this system it is necessary to fulfill the usual compatibility condition

$$
\operatorname{det}(D)=0
$$

The number of particles equations are obtained by using the expectation values of the $N_{p}, N_{n}$ operators on the vacuum $[16,30]$ :

$$
\begin{aligned}
Z= & w^{1}+x_{i j}^{1}\left\langle\mathcal{N}_{i j}\right\rangle \\
= & 2 \Omega\left(v_{1 p}^{2}+v_{2 p}^{2}\right)+\left(u_{1 p}^{2}-v_{1 p}^{2}\right)\left\langle\mathcal{N}_{11}\right\rangle+\left(u_{2 p}^{2}-v_{2 p}^{2}\right)\left\langle\mathcal{N}_{22}\right\rangle \\
& +\left(u_{1 p} u_{2 p}-v_{1 p} v_{2 p}\right)\left(\left\langle\mathcal{N}_{12}+\mathcal{N}_{21}\right\rangle\right) \\
N= & w^{2}+x_{i j}^{2}\left\langle\mathcal{N}_{i j}\right\rangle \\
= & 2 \Omega\left(v_{1 n}^{2}+v_{2 n}^{2}\right)+\left(u_{1 n}^{2}-v_{1 n}^{2}\right)\left\langle\mathcal{N}_{11}\right\rangle+\left(u_{2 n}^{2}-v_{2 n}^{2}\right)\left\langle\mathcal{N}_{22}\right\rangle \\
& +\left(u_{1 n} u_{2 n}-v_{1 n} v_{2 n}\right)\left(\left\langle\mathcal{N}_{12}+\mathcal{N}_{21}\right\rangle\right) .
\end{aligned}
$$

If the expectation value in Eq. (3.1) is taken over the standard BCS vacuum $|\mathrm{BCS}\rangle$ all matrix elements (me's) of the $\mathcal{N}$ and $\mathcal{P}$ operators vanish and one obtains the usual pn system of equations [24] in which only the first two terms in Eq. (3.2) have contribution. However, Eqs. (3.2) and (3.6) are also valid for a more general vacuum, as we will show below. The standard BCS system of equations (3.2) can be written as

$$
\begin{aligned}
& \epsilon_{p}^{\prime \prime}\left(v_{a p} u_{b p}+u_{a p} v_{b p}\right)+\epsilon_{n}^{\prime \prime}\left(v_{a n} u_{b p}+u_{a n} v_{b p}\right) \\
& +\Gamma_{p n}\left(v_{a p} u_{b n}+u_{a p} v_{p n}\right)+\Gamma_{p n}\left(v_{a n} u_{b p}+u_{a n} v_{b p}\right) \\
& -\Delta_{p p}\left(u_{a p} u_{b p}-v_{a p} v_{a p}\right)-\Delta_{n n}\left(u_{a n} u_{b n}-v_{a n} v_{a n}\right) \\
& -\Delta_{p n}\left(u_{a p} u_{b n}-v_{a n} v_{b p}\right)-\Delta_{p n}\left(u_{a n} u_{b p}-v_{a p} v_{b n}\right)=0,
\end{aligned}
$$

where the following notation was introduced: 


$$
\begin{gathered}
\epsilon_{p}^{\prime \prime}=\epsilon_{p}-G_{p} \rho_{p p}+\frac{F_{p n}}{4}-\frac{G_{p n}+F_{p n}}{2} \rho_{n n}, \\
\epsilon_{n}^{\prime \prime}=\epsilon_{n}-G_{n} \rho_{n n}+\frac{F_{p n}}{4}-\frac{G_{p n}+F_{p n}}{2} \rho_{p p}, \\
\Gamma_{p n}=F_{p n}^{\prime} \Omega \rho_{p n}, \\
\Delta_{p n}=G_{p n}^{\prime} \Omega \chi_{p n}, \\
\Delta_{p p}=G_{p} \Omega \chi_{p p}, \\
\Delta_{n n}=G_{p} \Omega \chi_{n n} .
\end{gathered}
$$

In this case the quasiparticle energies defined by Eq. (2.14) are automatically diagonal.

First of all let us discuss which are the possible cases to have solutions for the system (3.7). We will generalize the analysis performed in Ref. [23] to the Hamiltonian (2.7), containing arbitrary $\mathrm{p}-\mathrm{h}$ and $\mathrm{p}-\mathrm{p}$ terms. By using the trigonometric representation of the amplitudes (2.2) and also by adding and substracting Eqs. (3.7) with $(a, b)=(1,1),(2,2)$ one obtains the following system of equations:

$$
\begin{gathered}
\left(\epsilon_{p \alpha}+\epsilon_{n \beta}\right) \cos ^{2} \phi+\left(\epsilon_{n \alpha}+\epsilon_{p \beta}\right) \sin ^{2} \phi+\left(\Gamma_{\alpha}-\Gamma_{\beta}\right) \sin 2 \phi=0, \\
{\left[\left(\epsilon_{p \alpha}-\epsilon_{n \beta}\right) \cos ^{2} \phi+\left(\epsilon_{n \alpha}-\epsilon_{p \beta}\right) \sin ^{2} \phi+\left(\Gamma_{\alpha}+\Gamma_{\beta}\right) \sin 2 \phi\right]} \\
\times \cos 2 \psi+\left[-\epsilon_{\alpha \beta} \sin 2 \phi+2 \Gamma_{\alpha \beta} \cos 2 \phi\right] \sin 2 \psi=0,
\end{gathered}
$$

$$
\begin{aligned}
& \left(\epsilon_{p \alpha}-\epsilon_{n \beta}\right) \cos ^{2} \phi+\left(\epsilon_{n \alpha}-\epsilon_{p \beta}\right) \sin ^{2} \phi \\
& \left.\quad+\left(\Gamma_{\alpha}+\Gamma_{\beta}\right) \sin 2 \phi\right] \sin 2 \psi \\
& \quad-\left[-\epsilon_{\alpha \beta} \sin 2 \phi+2 \Gamma_{\alpha \beta} \cos 2 \phi\right] \cos 2 \psi=0,
\end{aligned}
$$

where we introduced the notation

$$
D(\alpha, \beta, \phi)=\left(\begin{array}{ccc}
\cos ^{2} \phi \sin 2 \alpha & \sin ^{2} \phi \sin 2 \alpha & -\left(\Delta_{p p} \cos ^{2} \phi+\Delta_{n n} \sin ^{2} \phi\right) \cos 2 \alpha+\Gamma_{\alpha} \sin 2 \phi \\
\sin ^{2} \phi \sin 2 \beta & \cos ^{2} \phi \sin 2 \beta & -\left(\Delta_{p p} \sin ^{2} \phi+\Delta_{n n} \cos ^{2} \phi\right) \cos 2 \beta-\Gamma_{\beta} \sin 2 \phi \\
\sin 2 \phi \sin (\alpha+\beta) & -\sin 2 \phi \sin (\alpha+\beta) & -\left(\Delta_{p p}-\Delta_{n n}\right) \sin 2 \phi \cos (\alpha+\beta)-2 \Gamma_{\alpha \beta} \cos 2 \phi
\end{array}\right)
$$

$$
\begin{gathered}
\epsilon_{\tau \alpha}=\epsilon_{\tau}^{\prime \prime} \sin 2 \alpha-\Delta_{\tau \tau} \cos 2 \alpha, \quad \tau=p, n, \\
\epsilon_{\tau \beta}=\epsilon_{\tau}^{\prime \prime} \sin 2 \beta-\Delta_{\tau \tau} \cos 2 \beta, \\
\epsilon_{\alpha \beta}=\left(\epsilon_{p}^{\prime \prime}-\epsilon_{n}^{\prime \prime}\right) \sin (\alpha+\beta)-\left(\Delta_{p p}-\Delta_{n n}\right) \cos (\alpha+\beta), \\
\Gamma_{\alpha}=\Gamma_{p n} \sin 2 \alpha-\Delta_{p n} \cos 2 \alpha, \\
\Gamma_{\beta}=\Gamma_{p n} \sin 2 \beta-\Delta_{p n} \cos 2 \beta, \\
\Gamma_{\alpha \beta}=\Gamma_{p n} \sin (\alpha+\beta)-\Delta_{p n} \cos (\alpha+\beta) .
\end{gathered}
$$

The last two equations (3.9) can be seen as a homogeneous linear system in $\sin \psi, \cos \psi$. One can obtain a nontrivial solution if the determinant of the coefficients vanishes; therefore the square brackets in Eq. (3.9) are separately vanishing. Finally the system of BCS equations does not depend on the variable $\psi$, which is consistent with the fact that the total energy (2.16) is also not dependent on this angle. By taking again the sum and difference of the first two equations one obtains

$$
\begin{gathered}
\epsilon_{p \alpha} \cos ^{2} \phi+\epsilon_{n \alpha} \sin ^{2} \phi+\Gamma_{\alpha} \sin 2 \phi=0, \\
\epsilon_{n \beta} \cos ^{2} \phi+\epsilon_{p \beta} \sin ^{2} \phi-\Gamma_{\beta} \sin 2 \phi=0, \\
\epsilon_{\alpha \beta} \sin 2 \phi-2 \Gamma_{\alpha \beta} \cos 2 \phi=0 .
\end{gathered}
$$

This system of equations is equivalent to the system of stationary conditions

$$
\frac{\partial H^{00}}{\partial \alpha}=\frac{\partial H^{00}}{\partial \beta}=\frac{\partial H^{00}}{\partial \phi}=0 .
$$

It can be seen as a system of three linear equations of the type (3.4) for the unknowns $\epsilon_{p}^{\prime \prime}, \epsilon_{n}^{\prime \prime}$ [or $\lambda_{p}, \lambda_{n}$ according to Eq. (2.8)]. In order to have nontrivial solutions it is necessary to fulfill the condition (3.5); i.e., the determinant of the matrix should vanish:

$$
\operatorname{det} D(\alpha, \beta, \phi)=0 \text {. }
$$

The angles $\alpha$ and $\beta$ are fixed by the number of particle conditions (3.6), which take the following form:

$$
\begin{aligned}
& \sin ^{2} \alpha \cos ^{2} \phi+\sin ^{2} \beta \sin ^{2} \phi=\frac{Z}{2 \Omega}, \\
& \sin ^{2} \alpha \sin ^{2} \phi+\sin ^{2} \beta \cos ^{2} \phi=\frac{N}{2 \Omega} .
\end{aligned}
$$



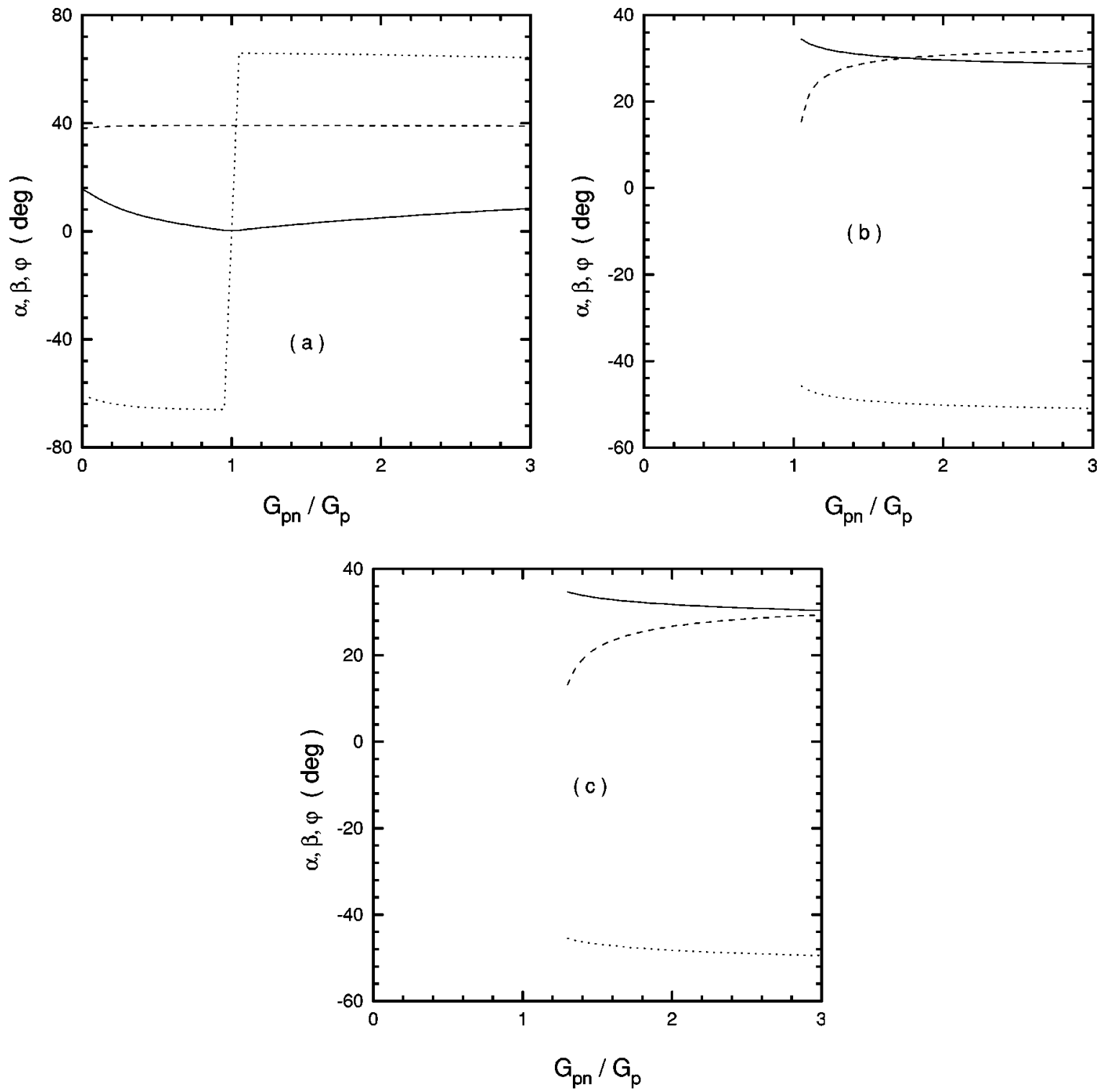

FIG. 1. (a) The angles $\alpha$ (solid line), $\beta$ (dotted line), and $\phi$ (dashed line) defined by Eq. (2.2) fulfilling the BCS compatibility condition defined by Eq. (3.14) versus the ratio $G_{p n} / G_{p}$ for the unstable regime. The parameters of the Hamitonian given by Eq. (2.7) are $G_{p}=G_{n}$ $=0.2, F_{p n}=0$, and $\Omega=6, Z=4, N=6$. (b) The same as in (a), but for the stable regime. (c) The same as in (b), but for $F_{p n} / G_{p}=2$.

We will show in the next section that the solutions (a1) and (a2) are stable against QRPA oscillations for complementary intervals of the $G_{p n}$ strength divided by a critical value $G_{c r t}$. Solution (a1) is stable for $G_{p n}<G_{c r t}$ and (a2) for $G_{p n}>G_{c r t}$.

The case $N \neq Z$ is important for the beta decay transitions. There are also two kinds of solutions.

(b1) The compatibility equation (3.14) as in the previous case has as solutions $\phi=0, \pi / 2$ and corresponds to an uncoupled system BCS equation for protons and neutrons, respectively. These solutions are stable for values of the particle-particle strength $G_{p n}<G_{c r t}$.

(b2) There is an important class of solutions with $\phi \neq 0$, $\psi=0$, corresponding to a coupled $p n$ system. Let us consider the following set of parameters: $G_{p}=G_{n}=0.2, F_{p n}$ $=0$. In Fig. 1(a) are shown the angles $\alpha$ (solid line), $\beta$ (dotted line), and $\phi$ (dashed line) for which the compatibility condition $\operatorname{det} D(Z, N, \phi)=0$ is fulfilled. There are two regions for which one has

$$
\begin{aligned}
& \text { (1) } G_{p n}<G_{c r t}, \quad \beta<0, \\
& \text { (2) } G_{p n}>G_{c r t}, \quad \beta>0 .
\end{aligned}
$$

These solutions are unstable with respect to the QRPA oscillations.

If the condition

$$
\text { (3) } G_{p n}>G_{c r t}, \quad \beta<0
$$

is fulfilled, as is shown in Fig. 1(b), then the solution becomes stable. Therefore the sign of the angle $\beta$, given as a square root according to Eq. (3.15), together with the value of the $G_{p n}$ strength determines the stability regime of the solution. 
From Fig. 1(a) one can see that for $G_{p n}=G_{c r t}$ the angle $\alpha$ vanishes. This critical case can be analytically analyzed. From Eq. (3.15) one obtains

$$
\sin ^{2} \beta=\frac{Z+N}{2 \Omega}, \quad \sin ^{2} \phi=\frac{Z}{Z+N}, \quad \cos ^{2} \phi=\frac{N}{Z+N} .
$$

The determinant of the matrix $D(Z, N, \phi)$ can easily be evaluated,

$$
\begin{aligned}
\operatorname{det} D(Z, N, \phi)= & \left(1-\frac{Z+N}{2 \Omega}\right)\left(G_{p}+G_{n}-2 G_{p n}^{\prime}\right) \\
& \times \sqrt{\frac{2}{\Omega}}\left(\frac{Z N}{Z+N}\right)^{3 / 2},
\end{aligned}
$$

and one obtains a vanishing value for two cases:

(a) $Z+N=2 \Omega$,

(b) $G_{p n}^{\prime}=\left(G_{p}+G_{n}\right) / 2$.

Case (b) is that given in Fig. 1(a). This value of the renormalized strength $G_{p n}^{\prime}$ is practically the critical value $G_{c r t}$. A similar conclusion for this particular case was drawn in Ref. [36].

The stability regime of the BCS solutions is connected with the exact exitation energy, given by the difference between the eigenvalues of the $(Z+1, N-1)$ and $(Z, N)$ systems, respectively. By increasing the $G_{p n}$ strength this energy difference of ground states changes its sign from plus to minus for $G_{p n}=G_{c r t}$, i.e., from a stable to an unstable regime.

For nonvanishing values of the $\mathrm{p}$-h strength $F_{p n}>0$ the situation remains qualitatively unchanged but the value $G_{c r t}$ is shifted to the right. This can be seen in Fig. 1(c), where the angles $\alpha, \beta, \phi$ fulfilling the compatibility condition (3.14) for $F_{p n} / G_{p}=2$ are plotted.
If one considers the SCQRPA vacuum, to be introduced below in the generalized BCS equation (3.2), the expectation values of the quasiparticle operators $\mathcal{N}, \mathcal{P}$ have nonvanishing contributions and, according to our calculations, the conclusions remain qualitatively unchanged; i.e., the compatibility condition (3.5) for a stable coupled $p n$ solution is also fulfilled for $G_{p n}>G_{c r t}, \beta<0$.

This concludes our study of the standard BCS solution of the Hamiltonian (2.12) when $p n(T=1)$ pairing is considered simultaneously with the $p p$ and $n n$ pairing.

Let us now come to the proper subject of this paper, namely, the study of extended RPA equations in this new quasiparticle basis introduced above.

\section{SELF-CONSISTENT QRPA EQUATIONS}

The extension of the RPA approach to include ground state correlations in a systematic way and which we called the self-consistent RPA (SCRPA) in the past [also called the cluster Hartree-Fock (CHF) approximation by other authors] seems to be a very powerful method to account for strong correlations in a Fermi system. These extensions have by now a quite long history and go back to Hara [37] who introduced already a renormalized RPA which can be considered as an approximation to the SCRPA. Later the idea of consistently accounting for ground state correlations in the RPA was much elaborated by Rowe [38]. More recently two of the present authors contributed more insight into the whole method in a series of papers $[14,17]$. Quite promising success for various models was achieved. The most relevant for our study here is the investigation with the SCRPA of a multilevel pairing model [39]. Naturally the SCRPA can also be extended to the superfluid case which we call the SCQRPA.

The SCRPA and SCQRPA can be derived from a variational principle. To this purpose we define the functional of an average excitation energy [34]

$$
E_{\mu}=\frac{\sum_{\nu k}\left\{\left(E_{\nu}-E_{0}\right)\left|\left\langle 0\left|X_{k}^{\mu} \mathcal{P}_{k}^{\dagger}\right| \nu\right\rangle\right|^{2}-\left(E_{\nu}-E_{0}\right)\left|\left\langle 0\left|Y_{k}^{\mu} \mathcal{P}_{k}\right| \nu\right\rangle\right|^{2}\right\}}{\sum_{\nu k}\left\{\left|\left\langle 0\left|X_{k}^{\mu} \mathcal{P}_{k}^{\dagger}\right| \nu\right\rangle\right|^{2}-\left|\left\langle 0\left|Y_{k}^{\mu} \mathcal{P}_{k}^{\dagger}\right| \nu\right\rangle\right|^{2}\right\}},
$$

where $|0\rangle$ and $|\nu\rangle$ are the ground and excited states and

$$
\mathcal{P}_{11}^{\dagger} \equiv \mathcal{P}_{1}^{\dagger}, \quad \mathcal{P}_{22}^{\dagger} \equiv \mathcal{P}_{2}^{\dagger}, \quad \mathcal{P}_{12}^{\dagger} \equiv \mathcal{P}_{3}^{\dagger}
$$

Minimization of $E_{\mu}$ with respect to $X_{k}^{\mu}, Y_{k}^{\mu}$ leads to

$$
\left\langle 0\left|\left[\delta \Gamma,\left[H, \Gamma_{\nu}^{\dagger}\right]\right]\right| 0\right\rangle=\omega_{\nu}\langle 0|\left[\delta \Gamma, \Gamma_{\nu}^{\dagger}|0\rangle,\right.
$$

where

$$
\Gamma_{\nu}^{\dagger}=\mathcal{P}_{k}^{\dagger} X_{k \nu}-\mathcal{P}_{k} Y_{k \nu}
$$

and $\delta \Gamma$ is a variation (with respect to $X, Y$ ) of $\Gamma$. It can also be shown that Eq. (4.3) is consistent with the condition

$$
\Gamma_{\nu}|0\rangle \equiv \Gamma_{\nu}|\mathrm{RPA}\rangle=0 ;
$$

that is, the ground state is the vacuum of the vibration annihilation operators $\Gamma_{\nu}$. More explicitly the SCQRPA equation (4.3) is written as

$$
\left(\begin{array}{ll}
\mathcal{A} & \mathcal{B} \\
\mathcal{B} & \mathcal{A}
\end{array}\right)\left(\begin{array}{l}
X \\
Y
\end{array}\right)=\left(\begin{array}{cc}
\mathcal{C} & 0 \\
0 & -\mathcal{C}
\end{array}\right)\left(\begin{array}{l}
X \\
Y
\end{array}\right) \omega,
$$


where $\omega$ is the diagonal matrix of the SCQRPA eigenvalues and $\mathcal{C}$ the metric matrix:

$$
\mathcal{C}_{i j}=\left\langle\left[\mathcal{P}_{i}, \mathcal{P}_{j}^{\dagger}\right]\right\rangle=\delta_{i j}-a_{i j}^{k}\left\langle\mathcal{N}_{k}\right\rangle, \quad i, j=1,2,3 .
$$

In the Appendix are given the detailed forms of the SCQRPA matrix elements. Equations (4.3)-(4.6) constitute in principle the SCQRPA equations. In the case when Eq. (4.5) can be explicitly solved for the ground state |RPA (see, e.g., [42]), one can calculate the matrix elements $\mathcal{A}, \mathcal{B}, \mathcal{C}$ as a function of $X, Y$ and find the solution of Eq. (4.6). Replacing in the evaluation of $\mathcal{A}, \mathcal{B}, \mathcal{C}$ the SCQRPA ground state by the BCS vacuum leads to the standard QRPA. In general it is very difficult to obtain the ground state from Eq. (4.5). In cases where it is not available, $\mathcal{A}, \mathcal{B}, \mathcal{C}$ can still be evaluated to a large extent making use of condition (4.5). Not all the elements of $\mathcal{A}, \mathcal{B}, \mathcal{C}$ can, however, be determined in this way and in general one has to apply some approximation.

It is also possible to include a fourth component in the basis

$$
\mathcal{P}_{4}^{\dagger}=\mathcal{N}_{12} \equiv \mathcal{N}_{3}
$$

and the diagonal metric matrix becomes

$$
\begin{gathered}
\mathcal{C}_{i j}=\delta_{i j}\left(1-\frac{\left\langle\mathcal{N}_{i}\right\rangle}{\Omega}\right), \quad i, j=1,2, \\
\mathcal{C}_{33}=1-\frac{\left\langle\mathcal{N}_{1}+\mathcal{N}_{2}\right\rangle}{2 \Omega}, \\
\mathcal{C}_{44}=\left\langle\mathcal{N}_{1}-\mathcal{N}_{2}\right\rangle .
\end{gathered}
$$

We will shortly recall the procedure to solve the SCQRPA equations in the most general case of a nondiagonal metric matrix. In order to solve the eigenvalue problem one first diagonalizes the metric matrix

$$
\mathcal{C} Z=Z \mu, \quad Z Z^{\dagger}=Z^{\dagger} Z=I,
$$

where $\mu$ is the diagonal eigenvalue metric matrix. By introducing the normalized eigenvectors

$$
\left(\begin{array}{c}
\bar{X} \\
\bar{Y}
\end{array}\right)=S\left(\begin{array}{c}
X \\
Y
\end{array}\right)
$$

and normalized matrices

$$
\left(\begin{array}{l}
\overrightarrow{\mathcal{A}} \\
\overline{\mathcal{B}}
\end{array}\right)=\left(S^{-1}\right)^{\dagger}\left(\begin{array}{l}
\mathcal{A} \\
\mathcal{B}
\end{array}\right) S^{-1},
$$

where

$$
S=\mu^{1 / 2} Z^{\dagger}, \quad S^{-1}=Z \mu^{-1 / 2},
$$

one obtains the SCQRPA system of equations in the same form as for the standard QRPA case:

$$
\left(\begin{array}{ll}
\overline{\mathcal{A}} & \overline{\mathcal{B}} \\
\overline{\mathcal{B}} & \overline{\mathcal{A}}
\end{array}\right)\left(\begin{array}{l}
\bar{X} \\
\bar{Y}
\end{array}\right)=\left(\begin{array}{cc}
I & 0 \\
0 & -I
\end{array}\right)\left(\begin{array}{l}
\bar{X} \\
\bar{Y}
\end{array}\right) \omega .
$$

In this way by introducing the vector $\mathcal{P}^{\dagger}=\left(\mathcal{P}_{k}^{\dagger}\right), \quad k$ $=1,2,3,4$, the SCQRPA phonon can be written as

$$
\Gamma^{\dagger}=\mathcal{P}^{\dagger} X-\mathcal{P} Y=\mathcal{P}^{\dagger} S^{-1} \bar{X}-\mathcal{P} S^{-1} \bar{Y} \equiv \overline{\mathcal{P}} \bar{X}-\overline{\mathcal{P} Y},
$$

where the normalized operators

$$
\overline{\mathcal{P}^{\dagger}}=\mathcal{P}^{\dagger} S^{-1}
$$

can be inverted:

$$
\overline{\mathcal{P}^{\dagger}}=\Gamma^{\dagger} \overline{X^{\dagger}}+\Gamma \overline{Y^{\dagger}} .
$$

With this inversion formula and using the vacuum condition (4.5) most of the terms in $\mathcal{A}, \mathcal{B}, \mathcal{C}$ can be evaluated with the explicit use of the wave function. Specifically this is done for the expectation values of the form $\left\langle\mathcal{P}^{\dagger} \mathcal{P}\right\rangle,\left\langle\mathcal{P} \mathcal{P}^{\dagger}\right\rangle,\left\langle\mathcal{P}^{\dagger} \mathcal{P}^{\dagger}\right\rangle$, and $\langle\mathcal{P P}\rangle$. However, the one-body densities $\left\langle\mathcal{N}_{i}\right\rangle$ and if one disregards the component $\mathcal{P}_{4}^{\dagger}$, as we will do here, also the elements $\left\langle\mathcal{N}_{i} \mathcal{N}_{j}\right\rangle$ cannot exactly be determined in terms of $X, Y$ without explicit knowledge of the wave function. However, there exists a fast converging expansion of $\mathcal{N}_{i}$ in terms of $\left(\mathcal{P}^{\dagger}\right)^{n}(\mathcal{P})^{n}$ [40] and then also the above expectation values can be calculated as a function of $X, Y$. An alternative way is to use the so-called number of particles operator technique $[38,41]$. In the present paper we will, as we did in our previous publications, restrict ourselves to the $\mathcal{P}_{3}^{\dagger} \equiv \mathcal{P}_{12}^{\dagger}$ component in the RPA pair creation operator (4.4); i.e., we only consider pair vibrations of the $p n$ type and we completely decouple it from the $p p$ and $n n$ vibrations. In this special case it is possible to construct the RPA ground state explicitely from Eq. (4.5). It has the same form as in our previous publication [30] and we will not repeat it here or give the explicit form for $\langle\mathcal{N}\rangle$ and $\langle\mathcal{N N}\rangle$ as we have already presented them in the Appendix of the above-mentioned paper. To neglect $p p$ and $n n$ components in the RPA operator (4.4) is certainly an approximation which must be released in the future. However, the appearance of spurious modes in the extended $3 \times 3$ version (see below) introduces nontrivial numerical and technical dificulties which go beyond the scope of this paper where we investigate the SCQRPA with only $p n$ mixing in the quasiparticle basis. The latter is so far open and we will determine it as usual from the minimization of the ground state energy. Having the ground state at hand we can calculate $\langle H\rangle$ and vary it with respect to the amplitudes $u, v$ of the quasiparticle transformation (2.1). We have shown elsewhere [14] that this procedure leads to the equations

$$
\left\langle\left[H, \mathcal{P}_{k}^{\dagger}\right]\right\rangle=0,
$$

which are analogous to Eqs. (3.2) but with the BCS ground state replaced by the SCQRPA ground state. It should be realized that Eq. (4.18) yields equations for $u, v$ which couple back to the RPA amplitudes. This is different from 
the standard BCS+RPA scheme where such a coupling is absent. It should also be realized that Eq. (4.18) together with the number equation (3.6) evaluated on the SCQRPA ground state ensures the fulfillment of the Ikeda sum rule as this was shown in our preceding work [16].

Before proceeding with numerical applications let us introduce the $\beta$ decay operators, since they are of greatest interest in the double beta decay process.

\section{BETA DECAY OPERATORS}

Let us introduce the monopole (Fermi) $\beta$ decay operators in the quasiparticle representation (2.9),

$$
\begin{aligned}
& T_{-}=N_{p n}=\mathcal{P}_{i j}^{\dagger} y_{i j}^{3}+\mathcal{P}_{i j} z_{i j}^{3}+\mathcal{N}_{i j} x_{i j}^{3} \equiv \mathcal{P}_{k}^{\dagger} R_{k}+\mathcal{P}_{k} T_{k}, \\
& T_{+}=N_{n p}=\mathcal{P}_{i j}^{\dagger} y_{i j}^{4}+\mathcal{P}_{i j} z_{i j}^{4}+\mathcal{N}_{i j} x_{i j}^{4} \equiv \mathcal{P}_{k}^{\dagger} T_{k}+\mathcal{P}_{k} R_{k},
\end{aligned}
$$

where the coefficients $x, y, z$ are defined in the Table I and the indices $k=1,2,3,4$ are those of the $\mathcal{P}$ operators defining the extended SCQRPA basis. Here we dropped the constant term which gives a vanishing contribution for transitions between different states. By inverting the $\mathcal{P}$ operators in terms of the SCQRPA phonons according to Eq. (4.17) one obtains for the transition matrix elements the general expression

$$
\begin{aligned}
& \left\langle\Gamma_{\mu}\left|T_{-}\right| 0\right\rangle=\overline{X_{\mu i}^{\dagger}} S_{i k} R_{k}+\overline{Y_{\mu i}^{\dagger}} S_{i k} T_{k}, \\
& \left\langle\Gamma_{\mu}\left|T_{+}\right| 0\right\rangle=\overline{X_{\mu i}^{\dagger}} S_{i k} T_{k}+\overline{Y_{\mu i}^{\dagger}} S_{i k} R_{k},
\end{aligned}
$$

where the matrix $S$ is defined by Eq. (4.13). Let us consider the difference giving the Ikeda sum rule for Fermi transitions,

$$
\sum_{\mu}\left|\left\langle\Gamma_{\mu}\left|T_{-}\right| 0\right\rangle\right|^{2}-\sum_{\mu}\left|\left\langle\Gamma_{\mu}\left|T_{+}\right| 0\right\rangle\right|^{2}=C_{i j}\left(R_{i} R_{j}-T_{i} T_{j}\right),
$$

where we used the orthonormality relation of the normalized SCQRPA amplitudes $\bar{X}, \bar{Y}$ and $C$ is the metric matrix. A straightforward calculation using the trigonometric representation of the BCS amplitudes (2.2) shows that the Ikeda sum rule

$$
\sum_{\mu}\left|\left\langle\Gamma_{\mu}\left|T_{-}\right| 0\right\rangle\right|^{2}-\sum_{\mu}\left|\left\langle\Gamma_{\mu}\left|T_{+}\right| 0\right\rangle\right|^{2}=N-Z
$$

is exactly fulfilled in two cases:

(1) if one considers all $k=1,2,3,4$ components in the SCQRPA phonon, or

(2) if one considers only the $k=3$ component but for $\psi$ $=0$.

The last variant is the case we considered in our paper because the coupled $p n$ solution reaches the energy minimum for $\psi=0$. In this case the summation in Eq. (5.4) is restricted to one term.

\section{NUMERICAL APPLICATION}

Let us first consider the exact solution of the Hamiltonian (2.7). In this case the wave functions of the system have a definite number of particles and are therefore given by the linear superposition [26]

$$
\begin{aligned}
|Z, N, \nu\rangle= & \sum_{Z=N_{0}+2 N_{1}, N=N_{0}+2 N_{2}} c_{N_{0} N_{1} N_{2}}(\nu) \\
& \times P_{p n}^{\dagger N_{0}} P_{p}^{\dagger N_{1}} P_{n}^{\dagger N_{2}}|0\rangle,
\end{aligned}
$$

where $P^{\dagger}$ operators are defined by Eq. (2.3) and $\nu$ labels the eigenstates given by the diagonalization procedure. The $T_{ \pm}$ operators connect $(Z, N)$ with $(Z \mp 1, N \pm 1)$.

Of course the Ikeda sum rule is automatically fulfilled:

$$
\begin{aligned}
\sum_{\nu}\left\langle Z, N, 0\left|N_{n p}\right| Z+1, N-1, \nu\right\rangle\left\langle Z+1, N-1, \nu\left|N_{p n}\right| Z, N, 0\right\rangle-\sum_{\nu}\left\langle Z, N, 0\left|N_{p n}\right| Z-1, N+1, \nu\right\rangle\left\langle Z-1, N+1, \nu\left|N_{n p}\right| Z, N, 0\right\rangle \\
\quad=\left\langle Z, N, 0\left|N_{n}\right| Z, N, 0\right\rangle-\left\langle Z, N, 0\left|N_{p}\right| Z, N, 0\right\rangle=N-Z .
\end{aligned}
$$

The energy of the $p n$ excitation is found as the difference between the eigenvalues for $(Z+1, N-1)$ and $(Z, N)$ nuclei.

As mentioned in Sec. IV, we only want to consider the $\mathcal{P}_{3}^{\dagger} \equiv \mathcal{P}_{12}^{\dagger}$ component of the QRPA operator

$$
\Gamma^{\dagger}=X \mathcal{P}_{3}^{\dagger}-Y \mathcal{P}_{3}
$$

An important point to be discussed is whether the excitation energy must be corrected for $\lambda_{p}-\lambda_{n}$ as ussualy done in the QRPA, in connection with the double beta decay (see, e.g., Ref. [30]). Also in Ref. [15] we applied a correction to the
SCRPA energy in order to account for the true ground state differences of the seniority model. We here argue that no correction of any of this kind should be applied because of the one-dimensional approximation (6.3) where the components $\mathcal{P}_{11}^{\dagger}$ and $\mathcal{P}_{22}^{\dagger}$ have been neglected. Indeed the latter two components correspond to spurious proton and neutron states, i.e., to rotation in the gauge space as we will discuss below. In a one-dimensional cut through a Mexican hat type of potential energy rotational motion is absent and then we also do not have to correct for it. These considerations will be confirmed by the study of the $3 \times 3$ QRPA, presented 
below, where we included $\mathcal{P}_{1}^{\dagger}, \mathcal{P}_{2}^{\dagger}, \mathcal{P}_{3}^{\dagger}$ components. We also will demonstrate that the one-dimensional SCQRPA is an appropriate approximation to the $3 \times 3$ case except in close vicinity of the transition point in the $p n$ interaction. Only in a rather narrow region around the phase transition does a coupling between the three modes exist. Our procedure is a straightforward generalization of the $p n$ states discussed in Refs. [16,30] where a BCS basis, which does not couple protons with neutrons, was used. In this case one can use the formulas for the SCQRPA vacuum in Ref. [30]. One also has the following relations:

$$
\begin{gathered}
\left\langle\mathcal{N}_{11}\right\rangle=\left\langle\mathcal{N}_{22}\right\rangle \equiv\langle\mathcal{N}\rangle, \\
\left\langle\mathcal{N}_{12}\right\rangle=\left\langle\mathcal{N}_{21}\right\rangle=0 .
\end{gathered}
$$

The SCQRPA system of equations (4.6) becomes a nonlinear equation to determine the eigenvalue $\omega$ and the amplitude $Y_{3}$. The conditions for the number of particles become, in this case,

$$
\begin{aligned}
& v_{1 p}^{2}+v_{2 p}^{2}=\sin ^{2} \alpha \cos ^{2} \phi+\sin ^{2} \beta \sin ^{2} \phi=\frac{Z-\langle\mathcal{N}\rangle}{2(\Omega-\langle\mathcal{N}\rangle)}, \\
& v_{1 n}^{2}+v_{2 n}^{2}=\sin ^{2} \alpha \sin ^{2} \phi+\sin ^{2} \beta \cos ^{2} \phi=\frac{N-\langle\mathcal{N}\rangle}{2(\Omega-\langle\mathcal{N}\rangle)},
\end{aligned}
$$

and allow us to express the angles $\alpha, \beta$ in terms of $\phi$. In order to have a solution of these equations it is necessary to fulfill the condition

$$
\langle\mathcal{N}\rangle \leqslant \min \{Z, N, 2 \Omega-Z, 2 \Omega-N\}
$$

By using the compatibility condition (3.5) together with the SCQRPA equation one expresses the Lagrange multipliers $\lambda_{p}, \lambda_{n}$ and the amplitude $Y_{3}$ as functions of two independent angles $\psi, \phi$. The criterion to select among different solutions is to find the constrained energy minimum of the system. This energy is calculated as the expectation value $\langle H\rangle$ of the Hamiltonian (2.12) on the SCQRPA vacuum. Our numerical analysis showed that, as in the pure BCS case, such a minimum is always reached for $\psi=0$ and therefore the angle $\phi$ is fully determined.

For the interaction strengths we selected the values $G_{p}$ $=G_{n}=0.2$ and $F_{p n} / G_{p}=0$ or 1 . The p-p strength $G_{p n}$, as usually in such a type of analysis, is taken as a free parameter on which depends the stability of the system. We also considered the degeneracy of the shell to be $\Omega=6$. This value was considered instead of $\Omega=5$ in our previous papers because it describes a more general nonsymmetric $p n$ system. We will analyze two important cases, namely,

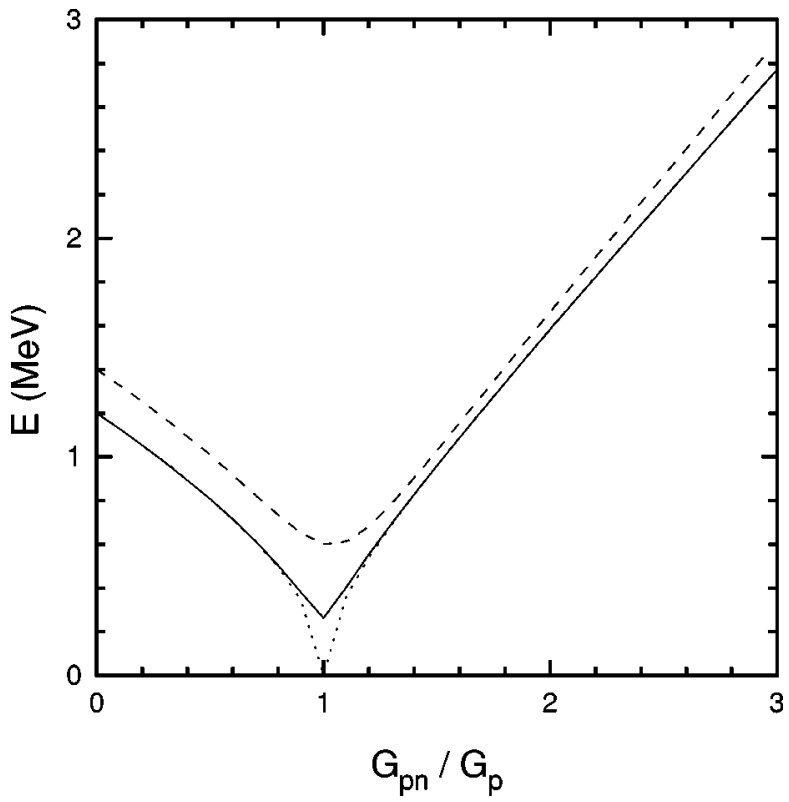

FIG. 2. The exact g.s. excitation energy (dashed line), the QRPA (dotted line), and the SCQRPA excitation energy (solid line) for $G_{p}=G_{n}=0.2, F_{p n}=0, \Omega=6, Z=N=4$.

$$
\text { A. } N=Z
$$

This case is very important because the system has exact g.s. stable (positive) solutions for any value of the p-p strength $G_{p n}$. Indeed in Fig. 2 is plotted by a dashed line the exact energy difference as a function of the $G_{p n} / G_{p}$ ratio for the case $N=Z=4$, with $F_{p n}=0$. It is computed as the difference between the ground states $(\nu=0)$ of the odd-odd $(Z+1, N-1)$ and even-even $(N, Z)$ nuclei using a diagonalization procedure described above. The difference is always positive and therefore the system is stable. This is also reflected by the QRPA analysis. The QRPA excitation energy is plotted in the same figure by a dotted line for two cases:

$$
\begin{gathered}
\text { (a) } G_{p n}<G_{c r t}, \quad \phi=0, \\
\text { (b) } G_{p n}>G_{c r t}, \quad \phi=\pi / 4 .
\end{gathered}
$$

Beyond the critical point $G_{c r t} / G_{p} \approx 1$ solution (a) becomes unstable (QRPA “breaks down"), but solution (b), corresponding to $\phi=\pi / 4$, becomes stable and follows the trend of the exact g.s. solution. In other words beyond this point the $p n$ uncoupled BCS basis with the angle $\phi=0$ is replaced by a coupled $p n$ basis, corresponding to a "deformed" minimum in the isospin space with $\phi=\pi / 4$. This is exactly the situation we want to clarify in this paper, i.e., how the QRPA or r-QRPA passes in a continuous way through the phase transition point. The QRPA excitation energy as a function of $G_{p n}$ is close to the exact energy, except for the region around the critical value $G_{c r t}$.

This conclusion is also confirmed by the analysis of the mean field stability matrix

$$
\mathcal{S}=\left(\begin{array}{ll}
\mathcal{A}_{i j} & \mathcal{P}_{i j} \\
\mathcal{P}_{i j} & \mathcal{A}_{i j}
\end{array}\right),
$$


whose components $i, j=1,2,3$ are computed in the Appendix. In this case all expectation values on the standard BCS vacuum (except $\left\langle\mathcal{P} P^{\dagger}\right\rangle$ ) vanish and the matrix (6.7) decouples into two blocks: a $4 \times 4$ block corresponding to the generators $\mathcal{P}_{1}^{\dagger}, \mathcal{P}_{2}^{\dagger}$ and a $2 \times 2$ block corresponding to the $p n$ $\mathcal{P}_{3}^{\dagger}$ component, whose eigenvalues $\nu_{k}, k=1,2$, are connected with the QRPA frequency by the following relation:

$$
\omega^{2}=\nu_{1} \nu_{2} .
$$

For the first solution (a) with $\phi=0$ before the critical value of the p-p strength $G_{p n}$ both eigenvalues $\nu_{k}$ are positive and the system is stable. Beyond the critical value $G_{c r t}, \nu_{1}$ becomes negative and therefore the QRPA frequency imaginary, the system becoming unstable. For any value of the $G_{p n}$ strength the four eigenvalues of the first block keep their sign constant: two of them are positive and two vanish, due to the number of particles conservation law. The second solution (b), with $\phi=\pi / 4$, displays an opposite picture. For $G_{p n}<G_{c r t}$ the system is unstable: at least one of the eigenvalues of the stability $2 \times 2$ submatrix is negative. Beyond this point both eigenvalues are positive and the system becomes stable.

Let us now consider the SCQRPA system of equations. As mentioned in Sec. III the BCS solutions should depend on angles $\psi, \phi$. For $\psi=0$ one obtains a minimum of the constrainded energy $\langle H\rangle$, defined as the expectation value of the Hamilonian (2.7) on the SCQRPA vacuum. In Fig. 2 is plotted by a solid line the SCQRPA solution versus the ratio $G_{p n} / G_{p}$. As in the QRPA case one has two kinds of solutions:

$$
\begin{aligned}
\left(\mathrm{a}^{\prime}\right) \quad G_{p n}<G_{c r t}, & \phi^{\prime}=0, \\
\left(\mathrm{~b}^{\prime}\right) \quad G_{p n}>G_{c r t}, & \phi^{\prime}=\pi / 4 .
\end{aligned}
$$

A significant difference with respect to the QRPA excitation energy occurs only around the critical value $G_{c r t} / G_{p}$ $\approx 1$. It is interesting to point out that the exact g.s. energy $E_{e e}$ of the even-even system $N=Z=4$, plotted in Fig. 3 by a dashed line, is close to the g.s. SCQRPA total energy $\langle\widetilde{H}\rangle$ (solid line) for $G_{p n}$ values before and after the phase transition. This energy is defined as the expectation value of the Hamiltonian (2.7), but without the Lagrange multipliers

$$
\langle\widetilde{H}\rangle \equiv\langle H\rangle+\lambda_{p} Z+\lambda_{n} N .
$$

\section{B. $N \neq Z$}

Let us consider the same set of parameters as in the previous case $G_{p}=G_{n}=0.2, F_{p n}=0, \Omega=6$, but with different number of protons and neutrons, respectively, $Z=4, N=6$. In Fig. 4 the QRPA excitation energy is shown as a function of the ratio $G_{p n} / G_{p}$ by a dotted line and the SCQRPA energy by a solid line. As in the previous case one has two kinds of solutions:

$$
\begin{aligned}
& \text { (a) } G_{p n}<G_{c r t}, \quad \phi=0, \\
& \text { (b) } G_{p n}>G_{c r t}, \quad \phi \neq 0,
\end{aligned}
$$

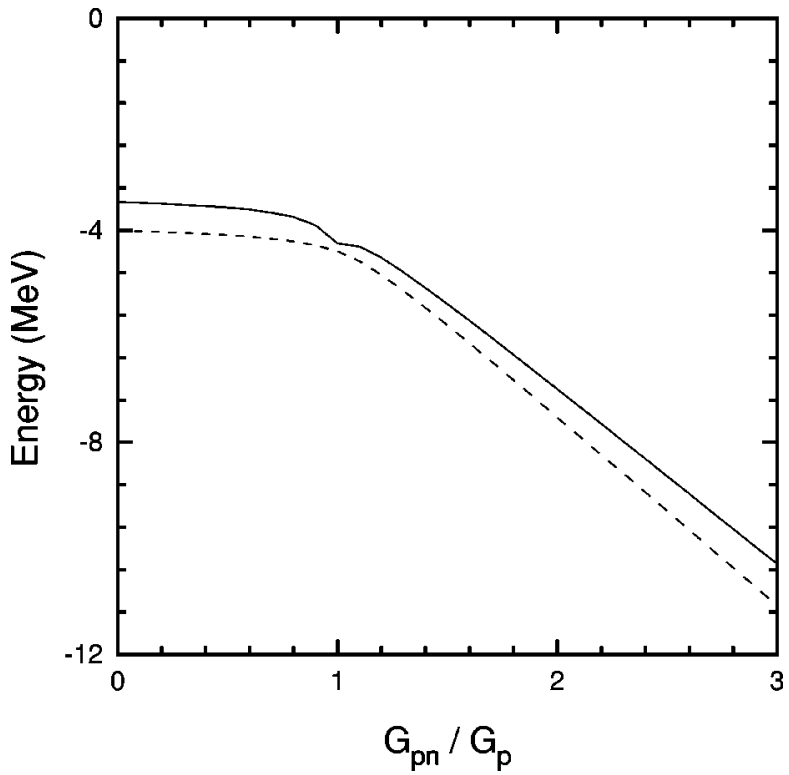

FIG. 3. The g.s. energy of the even-even nucleus (dashed line) and the expectation value of the Hamiltonian $\langle\tilde{H}\rangle$ given by Eq. (6.9) on the SCQRPA vacuum (solid line) versus the ratio $G_{p n} / G_{p}$. For $G_{p n} / G_{c r t}<1$ one has a $p n$ uncoupled solution with $\phi=0$ while for $G_{p n} / G_{c r t}>1$ a coupled $p n$ solution with $\phi=\pi / 4$. The parameters are the same as in Fig. 2.

where $G_{c r t} / G_{p} \approx 1$. The values of the angle $\phi$ for solution (b) are those in Fig. 1(b), corresponding to a stable $p n$ coupled BCS solution. The coupled $p n$ solution (b) beyond the critical point has a minimum with respect to the constrined energy $\langle H\rangle$ for the angle $\psi=0$.

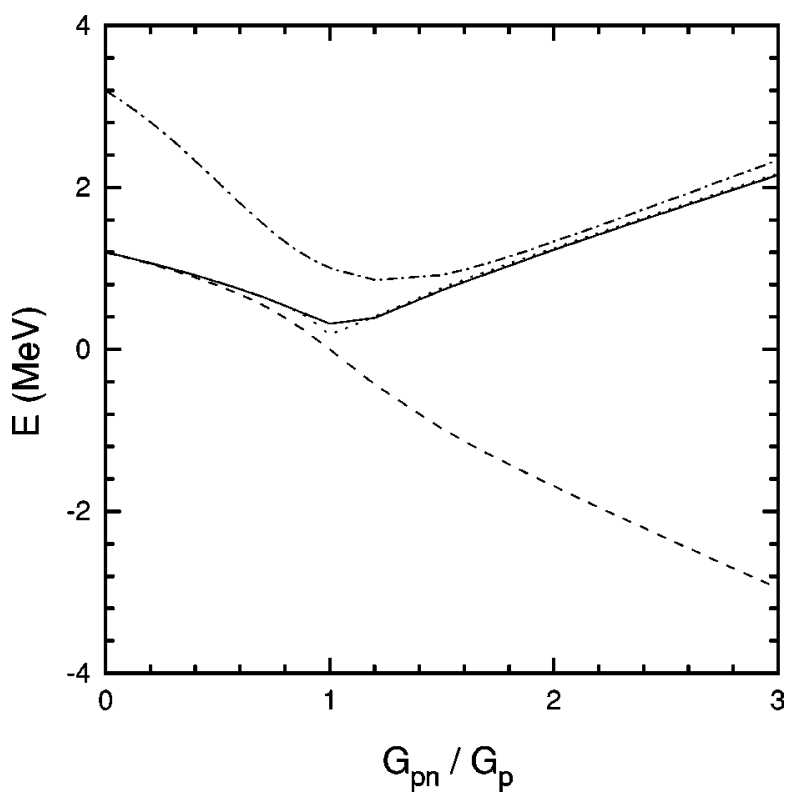

FIG. 4. The SCQRPA (solid line) and QRPA (dotted line) excitation energy versus the ratio $G_{p n} / G_{p}$ for $G_{p}=G_{n}=0.2, F_{p n}$ $=0, \Omega=6, Z=4, N=6$. For $G_{p n} / G_{c r t}<1$ one has an uncoupled $p n$ solution with $\phi=0$ while for $G_{p n} / G_{c r t}>1$ a coupled $p n$ solution with $\phi \neq 0$. The lower dashed line corresponds the the exact g.s. energy while the upper dot-dashed line to the second eigenvalue in the odd-odd nucleus. 


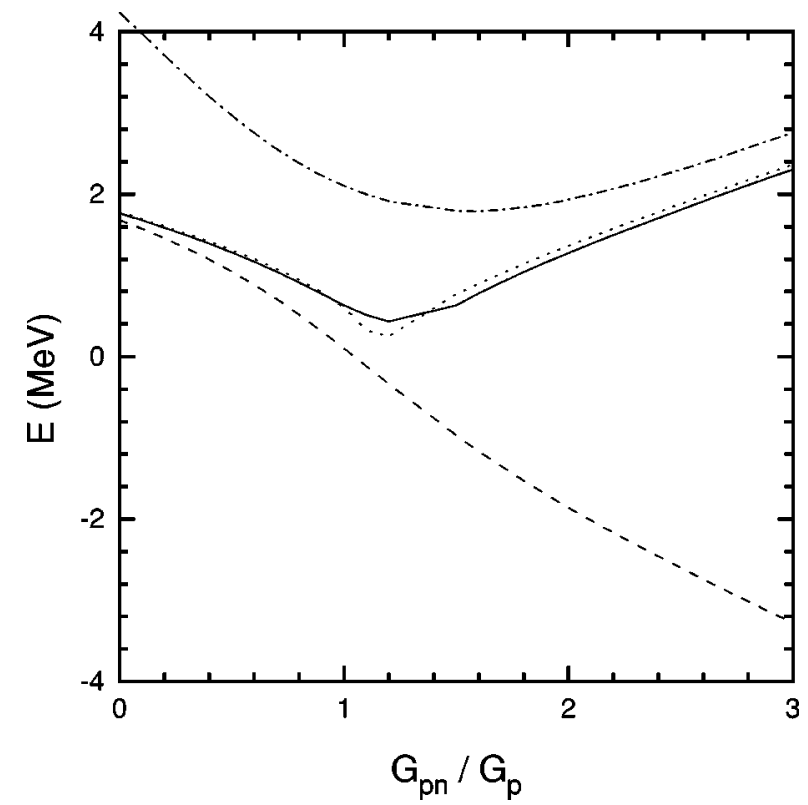

FIG. 5. The same as in Fig. 4 , but for $F_{p n} / G_{p}=1$.

The lower dashed line of Fig. 4 represents the exact difference of ground states $(\nu=0)$ equal to $E(Z+1, N-1)$ $-E(Z, N)$, whereas the upper dash-dotted line stands for the exact first excitation energy in the odd-odd $(Z+1, N-1)$ system $(\nu=1)$. It is obvious that the QRPA interpolates between these two energy differences. One must realize that the $N \neq Z$ situation is very different from the $N=Z$ case considered before. Indeed there the exact difference $E(Z+1, N$ $-1)-E(Z, N)$ was always positive and the QRPA is therefore able to follow with its eigenvalue the energy difference for all values of $G_{p n}$. This is no longer the case for the present $N \neq Z$ situation. Indeed the energy difference between $(Z+1, N-1)$ and $(Z, N)$ systems turns negative for $G_{p n} \approx G_{c r t}$. Therefore there is no possibility that the QRPA eigenvalue represents this energy difference for $G_{p n} \geqslant G_{c r t}$ in the $p n$ uncoupled basis.

If one includes the $p$-h interaction in the Hamiltonian (2.7), the situation remains qualitatively unchanged. In Fig. 5 we show by a dotted line the QRPA and by a solid line the SCQRPA excitation energy for the same set of parameters, but with $F_{p n} / G_{p}=1$. One can see that the critical strength $G_{c r t}$ is shifted to the right with respect to Fig. 4.

Let us come back to our restriction on the $\mathcal{P}_{12}^{\dagger}$ component only. At least at the QRPA level it is straightforward to also include the $\mathcal{P}_{11}^{\dagger}$ and $\mathcal{P}_{22}^{\dagger}$ components. In addition to the $p n$ pair vibration mode we then obtain two spurious QRPA solutions at zero energy, corresponding to particle number violation in $N$ and $Z$. The uncorrected $p n$ vibrational QRPA energy is shown in Fig. 6 by the upper solid line. However, as we argued above, in the $3 \times 3$ case with the spurious modes the rotational energy in the gauge space has to be corrected for when considering the $p n$ QRPA eigenvalue. This means that we have to substract from this eigenvalue the difference $\lambda_{p}-\lambda_{n}$ [30] and the result (lower solid line) then corresponds very closely to the exact excitation energy of the first excited state in the $Z+1, N-1$ system (dashed

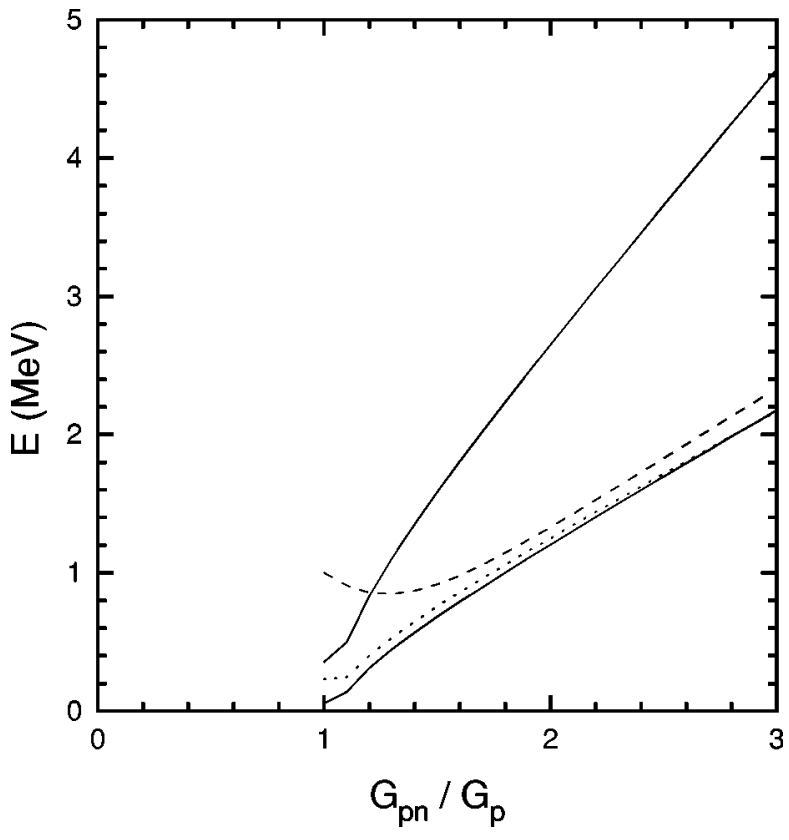

FIG. 6. The $p n$ excitation energy of the $3 \times 3$ QRPA (upper solid line), the corrected energy by the term $\lambda_{p}-\lambda_{n}$ (lower solid line), the one-component QRPA energy (dotted line), and the first excited exact solution (dashed line) versus the ratio $G_{p n} / G_{p}$ for the same parameters as in Fig. 4.

line). As we see it is also very close to the eigenvalue of the one-component QRPA (dotted line). We therefore conclude that the one-dimensional cut through the three-dimensional (rotational invariant) Mexican-hat-like surface, leading to the one-dimensional problem, yields a rather good approximation. Of course, as discussed before, no rotation (in gauge space) is possible in the one-dimensional reduction and therefore no correction $\lambda_{p}-\lambda_{n}$ has to be adopted in this case. This finding may hold true for other cases of spontaneous broken continuous symmetries, as well.

Let us now discuss the results concerning the beta decay matrix elements in the SCQRPA and exact cases. We present in Fig. 7(a) the me's for the exact solution of the beta decay operators $T_{-}$squared connecting the ground states of the even-even system $(Z, N)$ to the one of the odd-odd system $(Z+1, N-1)$, i.e., $\left|\left\langle Z+1, N-1 ; 0\left|T_{-}\right| Z, N ; 0\right\rangle\right|^{2}$ given by the solid line. This transition probability is given as a function of the ratio $G_{p n} / G_{p}$. The dashed line in Fig. 7(a) represents the SCQRPA transition me's squared $\left|\left\langle\Gamma\left|T_{-}\right| 0\right\rangle\right|^{2}$. We see that before the phase transition the SCQRPA reproduces quite well the exact solution.

After the phase transition point, where the $p n$ pairing catches on, the SCQRPA transition probability undershoots the exact values quite a bit. The deterioration of the agreement is not unexpected, since the situation is more complex after the phase transition. Indeed in this region we have an additional symmetry breaking where $p n$ pairing mixes eveneven and odd-odd ground states. In analogy with the static nonvanishing quadrupole moment of a deformed nucleus we here then have also a nonvanishing matrix element squared $\left|\left\langle 0\left|T_{-}\right| 0\right\rangle\right|^{2}$ after the phase transition. We show this by the solid curve in Fig. 7(b). In the exact case one could think that 

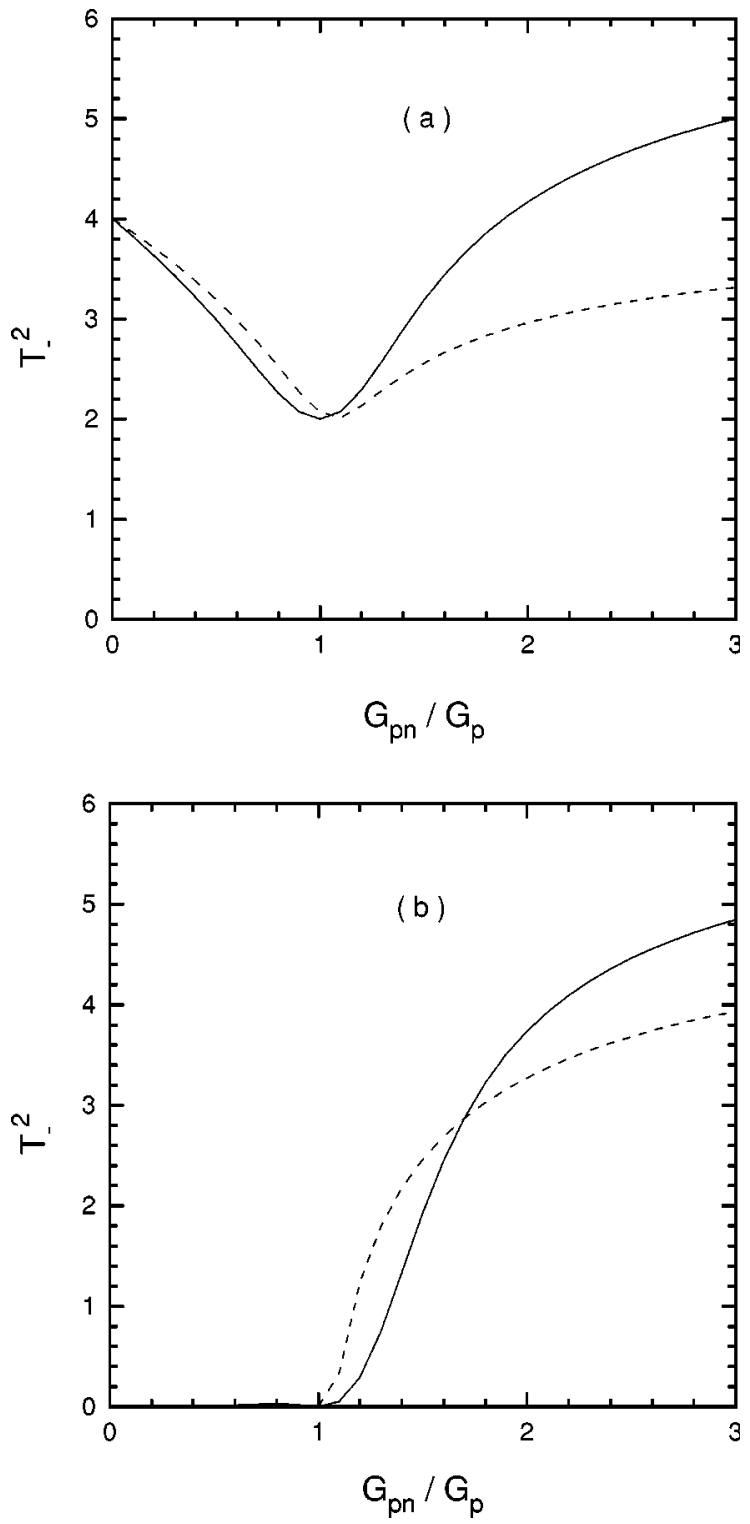

FIG. 7. (a) The beta decay matrix elements squared $\mid\langle Z+1, N$ $\left.-1,0\left|T_{-}\right| Z, N, 0\right\rangle\left.\right|^{2}$ (solid line), $\left|\left\langle\Gamma\left|T_{-}\right| 0\right\rangle\right|^{2}$ (dashed line). (b) The beta decay matrix elements squared $\left|\left\langle 0\left|T_{-}\right| 0\right\rangle\right|^{2}$ (solid line), $\mid\langle Z$ $\left.+1, N-1,1\left|T_{-}\right| Z, N, 0\right\rangle\left.\right|^{2}$ (dashed line).

the transition probability of the first excited state $\mid Z+1, N$ $-1 ; 1\rangle$ in the odd-odd system to the ground state in the even-even system could approximately follow the static moment $\left\langle 0\left|T_{-}\right| 0\right\rangle$ in the $p n$ deformed region. This is in analogy to a $\beta$-vibration transition probability in a deformed nucleus which follows the value of the static ground state quadrupole moment. We therefore also show in Fig. 7(b) by a dashed line the exact transition probability $\mid\langle Z+1, N-1 ; \nu$ $\left.=1\left|T_{-}\right| Z, N ; \nu=0\right\rangle\left.\right|^{2}$. We see that there is indeed an obvious correspondence between the two lines in Fig. 7(b) in spite of the fact that detailed agreement is seen to be only of a qualitative nature.

This probably stems from the fact that particle number is not a conserved quantity in our approach (besides the fact that the quantal fluctuation contained in the SCQRPA are able to restore particle number symmetry to a large extent, as shown in [15]). Indeed as we already said once the $p n$ pairing is nonvanishing in the region after the phase transition, even-even and odd-odd systems become mixed and it is very difficult to make any definite statement about transitions from one state to the other unless one has exact particle number projection.

Of course the Ikeda sum rule is exactly fulfilled for all values of the p-p strength $G_{p n}$.

\section{CONCLUSIONS}

In this paper we reanalyzed the schematic but rather general $\mathrm{O}(5)$ model representing the one-level proton-neutron isovector pairing Hamiltonian. This Hamiltonian was used in the past to investigate the QRPA solution in the region where the proton-neutron pair interation strength $G_{p n}$ is so strong that it provokes a collapse of the QRPA. The point we wanted to exploit in this work is based on the general wisdom [14] that once one arrives at a collapse of the RPA (or QRPA), then one has to change the single particle basis and continue with the RPA (or QRPA) beyond the phase transituion point using now the "deformed" basis. In the present case, since the collapse comes as a function of $G_{p n}$, one has to go from a BCS state which pairs $p p$ and $n n$ states separately to a more general BCS theory which in addition allows for $p n$ pairing. This is confirmed by a detailed stability study of the BCS ground state energy. Indeed we find that working with the more general BCS state including simultaneously $p p, n n$, and $p n$ pairing the corresponding QRPA shows a continuous crossover from before to after the critical value of $G_{p n}$. After the phase transition the $3 \times 3$ QRPA shows two spurious modes corresponding to the particle number breaking of protons and neutrons while there is one physical solution corresponding to a $p n$ pair vibrational state. We also showed that the QRPA eigenvalue has to be corrected for the "rotational energy" in gauge space as this was advocated long ago by Krmpotic et al. [30]. After having done this the QRPA physical eigenvalue shows indeed good agreement with the exact solution (Fig. 6). We also showed that once we reduce the QRPA to the one-dimensional case, i.e., including only $p n$ pairs in the RPA operator, then there is no need for correction since we hinder the system to "rotate." Still, as is demonstrated in Fig. 6, the one-dimensional noncorrected solution is in good agreement with the corrected three-dimensional solution. For the case of the SCQRPA we therefore restricted ourselves to the one-dimensional case, since the treatment of spurious motion is a delicate problem in this extended RPA theory [34]. We find that the SCQRPA improves the QRPA around the phase transition point. It also passes in a completely continuous way from before to after the critical point. The results for the $\beta$-transition matrix elements are more delicate to interpret. Before the critical $G_{p n}$ there is no problem and the various RPA results agree reasonably well with the exact solution. However, after the transition point the agreement is of a qualitative nature only. We attribute this to the fact that in the region with $p n$ pairing even-even and odd-odd systems become mixed and therefore it is very difficult to attribute $\beta$-transition matrix elements of the QRPA (or SCQRPA) to any transition between definite 
states of the exact solution. Any further progress is therefore probably only possible once one gets number projection under firm control.

We stress the fact that the Ikeda sum rule is fulfilled in the one-dimensional SCQRPA.

In our analysis for the sake of simplicity we negected the isoscalar component of the proton-neutron interaction. In any case it can only be important for $N \sim Z$ nuclei. A future in- clusion of the isoscalar pairing within the $\mathrm{O}(8)$ model will complete our analysis.

\section{ACKNOWLEDGMENTS}

One of us (D.S.D.) is grateful for the financial support given by CNRS during his stay in Grenoble, where the work was performed. Discussions with F. Simkovic are gratefully acknowledged.

\section{APPENDIX}

In order to derive the SCQRPA matrices for the generators given by Eqs. (4.2) let us introduce for the double commutators the notation

$$
\begin{gathered}
{\left[\mathcal{P}_{i},\left[\mathcal{P}_{j}, \mathcal{P}_{k}^{\dagger}\right]\right]=-c_{i b}^{a} a_{j k}^{b} \mathcal{P}_{a} \equiv-d_{i j k}^{a} \mathcal{P}_{a},} \\
{\left[\left[\mathcal{P}_{k}, \mathcal{P}_{j}^{\dagger}\right], \mathcal{P}_{i}^{\dagger}\right]=-d_{i j k}^{a} \mathcal{P}_{a}^{\dagger},} \\
{\left[\mathcal{P}_{i},\left[\mathcal{N}_{j}, \mathcal{P}_{k}^{\dagger}\right]\right]=b_{j k}^{i}-a_{i b}^{a} b_{j k}^{b} \mathcal{N}_{a} \equiv b_{j k}^{i}-e_{i j k}^{a} \mathcal{N}_{a},} \\
{\left[\left[\mathcal{P}_{i}, \mathcal{N}_{j}\right], \mathcal{P}_{k}^{\dagger}\right]=c_{i j}^{k}-a_{b k}^{a} c_{i j}^{b} \mathcal{N}_{a} \equiv c_{i j}^{k}-f_{i j k}^{a} \mathcal{N}_{a} .}
\end{gathered}
$$

The labels we used here are for pair indices: $i=1=(1,1), 2=(2,2), 3=(1,2), 4=(2,1)$ for $\mathcal{N}_{i}$ and $i=1=(1,1), 2=(2,2)$, $3=(1,2)$ for $\mathcal{P}_{i}$ operators. The terms in the Hamiltonian (2.12) giving the contribution for the double commutators are $H^{11}, H^{22}, H^{40}$. For symmetrized double commutators [38], giving the QRPA matrices for the pair indices $i, j=1,2,3$, one obtains the following relations:

$$
\begin{aligned}
\mathcal{A}_{i j} \equiv & \left\langle\left[\mathcal{P}_{i}, H, \mathcal{P}_{j}^{\dagger}\right]\right\rangle=\frac{1}{2} E_{n}\left[b_{n j}^{i}+c_{i n}^{j}-\left(e_{i n j}^{a}+f_{i n j}^{a}\right)\left\langle\mathcal{N}_{a}\right\rangle\right]+h_{i j}^{22}-\left(h_{m j}^{22} a_{i m}^{a}+h_{i n}^{22} a_{n j}^{a}\right)\left\langle\mathcal{N}_{a}\right\rangle+h_{m n}^{22} a_{i m}^{a} a_{n j}^{b}\left\langle\mathcal{N}_{a} \mathcal{N}_{b}\right\rangle \\
& -\frac{1}{2} h_{m n}^{22}\left(d_{i n j}^{a}\left\langle\mathcal{P}_{m}^{\dagger} B_{a}\right\rangle+d_{j m i}^{a}\left\langle\mathcal{P}_{a}^{\dagger} \mathcal{P}_{n}\right\rangle\right)+\frac{1}{2} g_{m n}^{22}\left[\left(b_{n j}^{i}+c_{i n}^{j}\right)\left\langle\mathcal{N}_{m}\right\rangle+\left(b_{m j}^{i}+c_{i m}^{j}\right)\left\langle\mathcal{N}_{n}\right\rangle-\left(e_{i n j}^{a}+f_{i n j}^{a}\right)\left\langle\mathcal{N}_{m} \mathcal{N}_{a}\right\rangle-\left(e_{i m j}^{a}+f_{i m j}^{a}\right)\right. \\
& \left.\times\left\langle\mathcal{N}_{a} \mathcal{N}_{n}\right\rangle+2 c_{i m}^{a} b_{n j}^{b}\left\langle\mathcal{P}_{a} \mathcal{P}_{b}^{\dagger}\right\rangle+2 b_{m j}^{a} c_{i n}^{b}\left\langle\mathcal{P}_{a}^{\dagger} \mathcal{P}_{b}\right\rangle\right]-\frac{1}{2} h_{m n}^{40}\left[d_{i n j}^{a}\left\langle\mathcal{P}_{m} \mathcal{P}_{a}\right\rangle+d_{i m j}^{a}\left\langle\mathcal{P}_{a} \mathcal{P}_{n}\right\rangle+d_{j n i}^{a}\left\langle\mathcal{P}_{m}^{\dagger} \mathcal{P}_{a}^{\dagger}\right\rangle+d_{j m i}^{a}\left\langle\mathcal{P}_{a}^{\dagger} \mathcal{P}_{n}^{\dagger}\right\rangle\right],(\mathrm{A} 2) \\
\mathcal{B}_{i j} \equiv & -\left\langle\left[\mathcal{P}_{i}, H, \mathcal{P}_{j}\right]\right\rangle=h_{i j}^{40}+h_{j i}^{40}-\frac{1}{2} h_{m n}^{22}\left(d_{i j m}^{a}+d_{j i m}^{a}\right)\left\langle\mathcal{P}_{a} \mathcal{P}_{n}\right\rangle-\left(h_{i n}^{40} a_{j n}^{a}+h_{m j}^{40} a_{i m}^{a}+h_{m i}^{40} a_{j m}^{a}+h_{j n}^{40} a_{i n}^{a}\right)\left\langle\mathcal{N}_{a}\right\rangle+h_{m n}^{40}\left(a_{i m}^{a} a_{j n}^{b}+a_{j m}^{a} a_{i n}^{b}\right) \\
& \times\left\langle\mathcal{N}_{a} \mathcal{N}_{b}\right\rangle-\frac{1}{2} h_{m n}^{40}\left[\left(d_{i j n}^{a}+d_{j i n}^{a}\right)\left\langle\mathcal{P}_{m}^{\dagger} \mathcal{P}_{a}\right\rangle+\left(d_{i j m}^{a}+d_{j i m}^{a}\right)\left\langle\mathcal{P}_{a} \mathcal{P}_{n}^{\dagger}\right\rangle\right]+g_{m n}^{22}\left(c_{i m}^{a} c_{j n}^{b}+c_{j m}^{a} c_{i n}^{b}\right)\left\langle\mathcal{P}_{a} \mathcal{P}_{b}\right\rangle .
\end{aligned}
$$

[1] J. Suhonen and O. Civitarese, Phys. Rep. 300, 123 (1998).

[2] P. Vogel and M. R. Zirnbauer, Phys. Rev. Lett. 57, 3148 (1986); D. Cha, Phys. Rev. C 27, 2269 (1987).

[3] J. Toivanen and J. Suhonen, Phys. Rev. Lett. 75, 410 (1995).

[4] J. Schwieger, F. Simkovic, and Amand Faessler, Nucl. Phys. A600, 179 (1996).

[5] F. Krmpotić, A. Mariano, E. J. V. de Passos, A. F. R. de Toledo Piza, and T. T. S. Kuo, Fiz. B 5, 93 (1996).

[6] F. Krmpotić, T. T. S. Kuo, A. Mariano, E. J. V. de Passos, and A. F. R. de Toledo Piza, Nucl. Phys. A612, 223 (1997).

[7] J. Toivanen and J. Suhonen, Phys. Rev. C 55, 2314 (1997).

[8] M. Sambataro and J. Suhonen, Phys. Rev. C 56, 782 (1997).
[9] F. Šimkovic, J. Schwieger, G. Pantis, and A. Faessler, Found. Phys. 27, 1275 (1997).

[10] K. Muto, Phys. Lett. B 391, 243 (1997).

[11] A. A. Raduta, C. M. Raduta, A. Faessler, and W. A. Kaminski, Nucl. Phys. A634, 497 (1998).

[12] F. Šimkovic, A. A. Raduta, M. Veselsky, and A. Faessler, Phys. Rev. C 61, 044319 (2000).

[13] R. V. Jolos and W. Rybarska-Nawrocka, Z. Phys. A 296, 73 (1980).

[14] J. Dukelsky and P. Schuck, Nucl. Phys. A512, 446 (1990); Mod. Phys. Lett. A 26, 2429 (1991).

[15] J. Dukelsky and P. Schuck, Phys. Lett. B 387, 233 (1996). 
[16] D. S. Delion, J. Dukelsky, and P. Schuck, Phys. Rev. C 55, 2340 (1997).

[17] J. Dukelsky, G. Röpke, and P. Schuck, Nucl. Phys. A628, 17 (1998).

[18] M. K. Cheoun, A. Bobyk, Amand Faessler, F. Šmkovic, and G. Teneva, Nucl. Phys. A561, 74 (1993); A564, 329 (1993).

[19] M. K. Cheoun, A. Faessler, F. Šimkovic, G. Teneva, and A. Bobyk, Nucl. Phys. A587, 301 (1995).

[20] O. Civitarese, M. Reboiro, and P. Vogel, Phys. Rev. C 56, 1840 (1997).

[21] B. Bremond and J. G. Valatin, Nucl. Phys. 41, 640 (1963).

[22] B. H. Flowers and M. Vujicic, Nucl. Phys. 49, 586 (1963).

[23] P. Camiz, A. Covello, and M. Jean, Nuovo Cimento Soc. Ital. Fis., B 42, 1839 (1966).

[24] A. Goswami, Nucl. Phys. 50, 228 (1964).

[25] A. L. Goodman, in Advances in Nuclear Physics, edited by J. W. Negele and E. Voigt (Plenum, New York, 1979), Vol. 11, p. 263.

[26] C. Dasso and A. Klein, Nucl. Phys. A210, 443 (1973).

[27] J. A. Evans, G. G. Dussel, E. E. Maqueda, and R. P. J. Perazzo, Nucl. Phys. A367, 77 (1981).

[28] G. G. Dussel, E. E. Maqueda, R. P. J. Perazzo, and J. A. Evans, Nucl. Phys. A450, 164 (1986).

[29] J. G. Hirsch, P. O. Hess, and O. Civitarese, Phys. Rev. C 54,
1976 (1996); Phys. Lett. B 390, 36 (1997); Phys. Rev. C 56, 199 (1997).

[30] F. Krmpotić, E. J. V. de Passos, D. S. Delion, J. Dukelsky, and P. Schuck, Nucl. Phys. A637, 295 (1998).

[31] G. Martinez-Pinedo, K. Lamganke, and P. Vogel, Nucl. Phys. A651, 379 (1999).

[32] D. R. Bes, O. Civitarese, E. E. Maqueda, and N. N. Scocola, Phys. Rev. C 61, 024315 (1999).

[33] J. Engel, S. Pittel, M. Stoitsov, P. Vogel, and J. Dukelsky, Phys. Rev. C 55, 1781 (1997).

[34] P. Ring and P. Schuck, The Nuclear Many Body Problem (Springer-Verlag, Berlin, 1980).

[35] W. Satula and R. Wyss, Phys. Lett. B 393, 1 (1997).

[36] P. Camiz, Nuovo Cimento A 40, 1220 (1964).

[37] K. Hara, Prog. Theor. Phys. 32, 88 (1964).

[38] D. J. Rowe, Rev. Mod. Phys. 40, 153 (1968); Nuclear Collective Motion (Methuen, London, 1970).

[39] J. Dukelsky and P. Schuck, Phys. Lett. B 464, 164 (1999).

[40] P. Schuck, in Proceedings of the International Conference on Clustering Aspects of Nuclear Structure and Dynamics, "Cluster '99,' Rab, Island of Rab, Croatia (World Scientific, Singapore, in press).

[41] P. Schuck and S. Ethofer, Nucl. Phys. A212, 269 (1973).

[42] A. A. Raduta, V. Ceausescu, and E. Badralexe, St. Cerc. Fiz. A28, 28 (1976). 